MATHEMATICS OF COMPUTATION

Volume 69, Number 230, Pages 629-652

S 0025-5718(99)01109-6

Article electronically published on August 24, 1999

\title{
BOUNDARY ELEMENT MONOTONE ITERATION SCHEME FOR SEMILINEAR ELLIPTIC PARTIAL DIFFERENTIAL EQUATIONS, PART II: QUASIMONOTONE ITERATION FOR COUPLED $2 \times 2$ SYSTEMS
}

\author{
GOONG CHEN, YUANHUA DENG, WEI-MING NI, AND JIANXIN ZHOU
}

\begin{abstract}
Numerical solutions of $2 \times 2$ semilinear systems of elliptic boundary value problems, whose nonlinearities are of quasimonotone nondecreasing, quasimonotone nonincreasing, or mixed quasimonotone types, are computed. At each step of the (quasi) monotone iteration, the solution is represented by a simple-layer potential plus a domain integral; the simple-layer density is then discretized by boundary elements. Because of the various combinations of Dirichlet, Neumann and Robin boundary conditions, there is an associated $2 \times 2$ matrix problem, the norm of which must be estimated. From the analysis of such $2 \times 2$ matrices, we formulate conditions which guarantee the monotone iteration a strict contraction staying within the close range of a given pair of subsolution and supersolution. Thereafter, boundary element error analysis can be carried out in a similar way as for the discretized problem. A concrete example of a monotone dissipative system on a $2 \mathrm{D}$ annular domain is also computed and illustrated.
\end{abstract}

\section{INTRODUCTION}

In an earlier paper [5], we have studied the boundary element method and monotone iteration scheme for semilinear elliptic boundary value problems with a single scalar governing equation. Tight error estimates were obtained for problems whose nonlinearity satisfies an assumption $[H]$. Numerical solutions for several concrete examples on a circular domain were computed, showing that they agree with the theoretical properties of stable solutions. In this paper, we extend our previous work to cover $2 \times 2$ systems of coupled semilinear elliptic boundary value problems.

Systems of coupled nonlinear elliptic equations arise frequently in applications. Solutions of such systems may be regarded as steady states of certain reactiondiffusion systems or problems in mathematical biology. For nonlinear problems, we face the ever present difficulties associated with existence/nonexistence, uniqueness/multiplicity and stability/instability of solutions. Since the nonlinearity is now further coupled and may appear somewhat arbitrary, the treatment is naturally

Received by the editor July 15, 1996 and, in revised form, April 30, 1998.

1991 Mathematics Subject Classification. Primary 31B20, 35J65, 65N30.

Key words and phrases. Nonlinear elliptic systems, boundary elements, error analysis, LotkaVolterra models.

Professors Chen and Zhou were supported in part by NSF Grants DMS 9404380 and 9610076. Professor Ni was supported in part by NSF Grants DMS 9401333 and 9705639. 
expected to be more complex and challenging than the case of a single scalar semilinear elliptic equation and, thus, many more problems remain open [9]. Though inasmuch as the mathematical intractability for coupled semilinear elliptic systems is more severe, it is fortunate that a satisfactory method for finding stable solutions for a certain class of such systems has been developed. This class consists of coupled systems whose nonlinearities are of the quasimonotone nondecreasing, quasimonotone nonincreasing, or mixed quasimonotone types. The method is a generalization of the monotone iteration scheme that we employed in Part I 5]. A good reference can be found in Pao [1]. Since the method is constructive, it can be discretized and implemented numerically by the established methods of finite difference, finite element and boundary element. Obviously, researchers in science and engineering have computed many examples of coupled semilinear elliptic (or parabolic) systems arising from applications, with or without the knowledge that the quasimonotone iteration scheme can be incorporated in their algorithms. In Pao [10], e.g., a finite difference-monotone iteration method is used to solve some coupled elliptic systems numerically. Nevertheless, we have not yet seen any global error analysis results in the literature. In particular, when the boundary element method (BEM) is used, rates of convergence with respect to the discretization parameter have never been analyzed for coupled elliptic systems, to the best of our knowledge. The crux of this paper, therefore, is to carry out the boundary element numerical analysis and computation for coupled semilinear elliptic systems using the quasimonotone iteration scheme.

A coupled semilinear elliptic system of boundary value problems may be likened to a $2 \times 2$ system of nonlinear matrix equations in an infinite dimensional space. The major contribution of this paper virtually is to exploit this $2 \times 2$ matrix structure of the PDE system, and formalize it to an infinite-dimensional setting. This is carried out in 92 and \&4. A critical assumption, $\left[H_{2 \times 2}^{2}\right]$, is stated, which is a generalization of our assumption $[H]$ in [5]. This $\left[H_{2 \times 2}^{2}\right]$ condition is directly verifiable and provides the basis of the local contraction argument in the permissible range of (quasi) monotone iteration. Once $\left[\mathrm{H}_{2 \times 2}^{2}\right]$ is well formulated, the rest of the error estimation procedures are pretty much the same as in [5]. We state the error estimates for the Galerkin boundary-element iterates $u_{h}^{n}(x)$ in the $H^{r}(\Omega), 0 \leq r<2$, Sobolev space norms in $\$ 3$. The discussion in $\$ 3$ will be concise, however, because as we have just said, the arguments are similar to [5]. In \$5, we provide a concrete example of a mixed quasimonotone $2 \times 2$ elliptic system for computation and discussion.

\section{Monotone iteration SCHEME FOR NONLINEARITIES \\ OF QUASIMONOTONE NONINCREASING, QUASIMONOTONE NONDECREASING, AND MIXED QUASIMONOTONE TYPES}

Let $\Omega$ be a simply connected bounded domain in $\mathbb{R}^{N}, N=2$ or 3 , with a smooth boundary $\partial \Omega$. (In much of the analysis, $\partial \Omega$ being $C^{2}$-continuous suffices.) We consider a coupled $2 \times 2$ system of semilinear elliptic partial differential equations of the form

$$
(S)\left\{\begin{array}{l}
\Delta u_{i}(x)=f_{i}\left(x, u_{1}(x), u_{2}(x)\right), x \in \Omega, \\
B_{i} u_{i}(x)=g_{i}(x), x \in \partial \Omega, \\
i=1,2,
\end{array}\right.
$$


where $B_{i}, i=1,2$, are linear boundary operators given by

$$
B_{i} u_{i}=u_{i} \quad \text { (Dirichlet type) }
$$

or

$$
B_{i} u_{i}=\frac{\partial u_{i}}{\partial n}+\alpha(\cdot) u_{i}, \quad \alpha(x) \geq 0 \text { on } \partial \Omega, \alpha \in C^{\infty}(\partial \Omega),
$$

(Neumann or Robin types)

and $f_{i} \in C^{\infty}(\Omega \times \mathbb{R} \times \mathbb{R}), g_{i} \in C^{\infty}(\partial \Omega)$, for $i=1,2$.

Throughout the paper, for $\Sigma=\Omega$ or $\partial \Omega$, we use $\|u\|_{s, \Sigma}$ to denote the norm of a scalar function $u$ in the Sobolev space $H^{s}(\Sigma)$, and use $\left\|\left(u_{1}, u_{2}\right)\right\|_{s, \Sigma}$ to denote the norm of a vector-valued function $\left(u_{1}, u_{2}\right)$ in the product Sobolev space $H^{s}(\Sigma) \times$ $H^{s}(\Sigma)$ :

$$
\left\|\left(u_{1}, u_{2}\right)\right\|_{s, \Sigma}^{2} \equiv\left\|u_{1}\right\|_{s, \Sigma}^{2}+\left\|u_{2}\right\|_{s, \Sigma}^{2} .
$$

It is well understood that the monotone iteration scheme for scalar semilinear elliptic boundary value problems has been generalized to system (S); see Pao [11, for example. For $(\underline{S})$, the construction of monotone iterates depends on the socalled quasimonotone properties of the reaction functions $f_{1}$ and $f_{2}$ in (S). Let $J=\left[a_{1}, b_{1}\right] \times\left[a_{2}, b_{2}\right] \subset \mathbb{R}^{2}$, for some $a_{i}, b_{i}, i=1,2$, satisfying $a_{i}<b_{i}$. A vectorvalued function $\left(f_{1}\left(x, u_{1}, u_{2}\right), f_{2}\left(x, u_{1}, u_{2}\right)\right)$ is called quasimonotone nonincreasing in $\left(x, u_{1}, u_{2}\right) \in \Omega \times J$, if

$$
\frac{\partial f_{1}}{\partial u_{2}}\left(x, u_{1}, u_{2}\right) \leq 0, \quad \frac{\partial f_{2}}{\partial u_{1}}\left(x, u_{1}, u_{2}\right) \leq 0, \quad\left(x, u_{1}, u_{2}\right) \in \Omega \times J ;
$$

quasimonotone nondecreasing in $\left(x, u_{1}, u_{2}\right) \in \Omega \times J$, if

$$
\frac{\partial f_{1}}{\partial u_{2}}\left(x, u_{1}, u_{2}\right) \geq 0, \quad \frac{\partial f_{2}}{\partial u_{1}}\left(x, u_{1}, u_{2}\right) \geq 0, \quad\left(x, u_{1}, u_{2}\right) \in \Omega \times J ;
$$

and mixed quasimonotone in $\left(x, u_{1}, u_{2}\right) \in \Omega \times J$, if

$$
\frac{\partial f_{1}}{\partial u_{2}}\left(x, u_{1}, u_{2}\right) \geq 0, \quad \frac{\partial f_{2}}{\partial u_{1}}\left(x, u_{1}, u_{2}\right) \leq 0, \quad\left(x, u_{1}, u_{2}\right) \in \Omega \times J .
$$

A pair of vector-valued functions $\left(\underline{u}_{1}, \underline{u}_{2}\right)$ and $\left(\bar{u}_{1}, \bar{u}_{2}\right)$ with $\underline{u}_{i}, \bar{u}_{i} \in C(\bar{\Omega}) \cap C^{2}(\Omega)$, $i=1,2$, are called ordered subsolution and supersolution, respectively, if they satisfy $\underline{u}_{i} \leq \bar{u}_{i}, i=1,2$, and

$$
B_{i} \underline{u}_{i}-g_{i} \leq 0, \quad B_{i} \bar{u}_{i}-g_{i} \geq 0, \quad \text { on } \partial \Omega, \quad i=1,2,
$$

plus

(i) for quasimonotone nonincreasing $\left(f_{1}, f_{2}\right)$,

$$
\begin{cases}\Delta \underline{u}_{1}-f_{1}\left(x, \underline{u}_{1}, \underline{u}_{2}\right) \geq 0, & \Delta \underline{u}_{2}-f_{2}\left(x, \underline{u}_{1}, \underline{u}_{2}\right) \geq 0, \\ \Delta \bar{u}_{1}-f_{1}\left(x, \bar{u}_{1}, \bar{u}_{2}\right) \leq 0, & \Delta \bar{u}_{2}-f_{2}\left(x, \bar{u}, \bar{u}_{2}\right) \leq 0 ;\end{cases}
$$

(ii) for quasimonotone nondecreasing $\left(f_{1}, f_{2}\right)$,

$$
\left\{\begin{aligned}
\Delta \underline{u}_{1}-f_{1}\left(x, \underline{u}_{1}, \bar{u}_{2}\right) \geq 0, & \Delta \underline{u}_{2}-f_{2}\left(x, \bar{u}_{1}, \underline{u}_{2}\right) \geq 0, \\
\Delta \bar{u}_{1}-f_{1}\left(x, \bar{u}_{1}, \underline{u}_{2}\right) \leq 0, & \Delta \bar{u}_{2}-f_{2}\left(x, \underline{u}_{1}, \bar{u}_{2}\right) \leq 0 ;
\end{aligned}\right.
$$

(iii) for mixed quasimonotone $\left(f_{1}, f_{2}\right)$,

$$
\left\{\begin{aligned}
\Delta \underline{u}_{1}-f_{1}\left(x, \underline{u}_{1}, \bar{u}_{2}\right) \geq 0, & \Delta \underline{u}_{2}-f_{2}\left(x, \underline{u}_{1}, \underline{u}_{2}\right) \geq 0, \\
\Delta \bar{u}_{1}-f_{1}\left(x, \bar{u}_{1}, \underline{u}_{2}\right) \leq 0, & \Delta \bar{u}_{2}-f_{2}\left(x, \bar{u}_{1}, \bar{u}_{2}\right) \leq 0 .
\end{aligned}\right.
$$


Henceforth, we assume that $\lambda_{i}, i=1,2$, are chosen sufficiently large such that

$$
\left[H_{2 \times 2}^{1}\right]\left\{\begin{array}{l}
(\mathrm{i}) \frac{\partial f_{i}\left(x, u_{1}, u_{2}\right)}{\partial u_{i}} \leq \lambda_{i}, \quad \forall\left(x, u_{1}, u_{2}\right) \in \Omega \times J, \quad i=1,2 ; \lambda_{i} \geq 0 ; \\
\text { (ii) the spectra of the operators }\left(\lambda_{i}-\Delta, B_{i}\right), i=1,2, \\
\quad \text { entirely lie on the open right half plane. }
\end{array}\right.
$$

Let

$$
\lambda_{i}^{*}=\text { the smallest eigenvalue of the operator }\left(-\Delta, B_{i}\right), \quad i=1,2 .
$$

Then obviously

$$
\left\{\begin{array}{l}
\lambda_{i}^{*} \geq 0, \quad i=1,2 ; \\
\lambda_{i}^{*}=0 \text { iff } B_{i} \text { corresponds to the homogeneous } \\
\text { Neumann boundary condition. }
\end{array}\right.
$$

From $\left[H_{2 \times 2}^{1}\right](\mathrm{i})$ and (ii), we see that the following iteration scheme is well-defined: for any initial state $\left(u_{1}^{(0)}, u_{2}^{(0)}\right)$ with $u_{i}^{(0)} \in C(\bar{\Omega}) \cap C^{2}(\Omega), i=1,2$,

$$
\left\{\begin{array}{l}
\left(\lambda_{i}-\Delta\right) u_{i}^{(n+1)}=F_{i, \lambda_{i}}\left(x, u_{1}^{(n)}, u_{2}^{(n)}\right), \quad \text { in } \Omega, \\
B_{i} u_{i}^{(n+1)}=g_{i}, \quad \text { on } \partial \Omega, \\
i=1,2, \text { and } n=0,1,2, \ldots,
\end{array}\right.
$$

where

$$
F_{i, \lambda_{i}}\left(x, u_{1}, u_{2}\right) \equiv \lambda_{i} u_{i}-f_{i}\left(x, u_{1}, u_{2}\right), \quad i=1,2 .
$$

The convergence of the scheme (2.15) and the monotonicity properties of the iterates depend on the choice of the initial state $\left(u_{1}^{(0)}, u_{2}^{(0)}\right)$, which in turn depends on the quasimonotone type of $\left(f_{1}, f_{2}\right)$. Let us proceed according to the recipe given in [11, §8.4]:

(i) For quasimonotone nonincreasing $\left(f_{1}, f_{2}\right)$ in $\Omega \times J$, let

$$
\left(u_{1}^{(0)}, u_{2}^{(0)}\right)=\left(\underline{u}_{1}, \underline{u}_{2}\right) \quad\left(\text { resp. }\left(\bar{u}_{1}, \bar{u}_{2}\right)\right) .
$$

We denote by $\left(\underline{u}_{1}^{(n)}, \underline{u}_{2}^{(n)}\right)\left(\right.$ resp. $\left.\left(\bar{u}_{1}^{(n)}, \bar{u}_{2}^{(n)}\right)\right), n=1,2, \ldots$, the corresponding iterates from (2.15).

(ii) For quasimonotone nondecreasing $\left(f_{1}, f_{2}\right)$ in $\Omega \times J$, let

$$
\left(u_{1}^{(0)}, u_{2}^{(0)}\right)=\left(\bar{u}_{1}, \underline{u}_{2}\right) \quad\left(\operatorname{resp} . \quad\left(\underline{u}_{1}, \bar{u}_{2}\right)\right) .
$$

We denote by $\left(\bar{u}_{1}^{(n)}, \underline{u}_{2}^{(n)}\right)\left(\operatorname{resp} .\left(\underline{u}_{1}^{(n)}, \bar{u}_{2}^{(n)}\right)\right), n=1,2, \ldots$, the corresponding iterates.

(iii) For mixed quasimonotone $\left(f_{1}, f_{2}\right)$ in $\Omega \times J$, the initial state is chosen as in (2.17), but the iteration procedure (2.15) is modified to

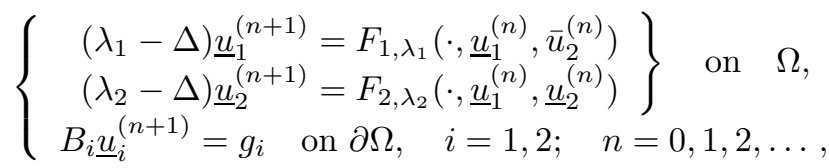

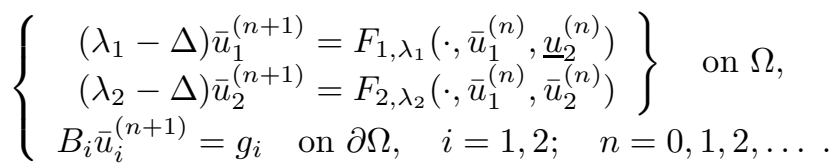


Following [1], (4.7), p. 403], we define the sector

$$
\langle\underline{\mathbf{u}}, \overline{\mathbf{u}}\rangle=\left\{\left(u_{1}, u_{2}\right) \mid u_{i} \in C(\bar{\Omega}), \quad \underline{u}_{i} \leq u_{i} \leq \bar{u}_{i}, \quad i=1,2\right\} .
$$

We now have the following convergence theorem.

Theorem 2.1. For each type of monotonicity, the sequences $\underline{u}_{i}^{(n)}, \bar{u}_{i}^{(n)}$, described in (2.15) -2.20) satisfy the monotone property

$$
\underline{u}_{i}^{(n)} \leq \underline{u}_{i}^{(n+1)} \leq \bar{u}_{i}^{(n+1)} \leq \bar{u}_{i}^{(n)} \quad \text { on } \bar{\Omega}, \quad i=1,2, \quad n=0,1,2, \ldots,
$$

and there exist functions $\underline{u}_{i}^{(\infty)}$ and $\bar{u}_{i}^{(\infty)}, i=1,2$, satisfying $\underline{u}_{i}^{(\infty)} \leq \bar{u}_{i}^{(\infty)}$ on $\bar{\Omega}$ such that $\underline{u}_{i}^{(n)}$ and $\bar{u}_{i}^{(n)}$ converge monotonically to $\underline{u}_{i}^{(\infty)}$ and $\bar{u}_{i}^{(\infty)}$, respectively, for $i=1,2$. We have

(i) if $\left(f_{1}, f_{2}\right)$ is quasimonotone nonincreasing, then $\left(\underline{u}_{1}^{(\infty)}, \underline{u}_{2}^{(\infty)}\right)$ and $\left(\bar{u}_{1}^{(\infty)}\right.$, $\left.\bar{u}_{2}^{(\infty)}\right)$ are solutions of $(\underline{S})$;

(ii) if $\left(f_{1}, f_{2}\right)$ is quasimonotone nondecreasing, then $\left(\underline{u}_{1}^{(\infty)}, \bar{u}_{2}^{(\infty)}\right)$ and $\left(\bar{u}_{1}^{(\infty)}\right.$, $\left.\underline{u}_{2}^{(\infty)}\right)$ are solutions of $(\underline{S})$;

(iii) if $\left(f_{1}, f_{2}\right)$ is mixed quasimonotone and satisfies

$$
\max \left\{\frac{\partial f_{i}\left(x, u_{1}, u_{2}\right)}{\partial u_{i}} \mid\left(x, u_{1}, u_{2}\right) \in \Omega \times\langle\underline{\mathbf{u}}, \overline{\mathbf{u}}\rangle\right\}<\lambda_{i}^{*}
$$

for either $i=1$ or $i=2$, then $\left(\underline{u}_{1}^{(\infty)}, \underline{u}_{2}^{(\infty)}\right)$ and $\left(\bar{u}_{1}^{(\infty)}, \bar{u}_{2}^{(\infty)}\right)$ are solutions of (S).

Moreover, any solution $\left(u_{1}, u_{2}\right)$ in $\langle\underline{\mathbf{u}}, \overline{\mathbf{u}}\rangle$ satisfies $\underline{u}_{i}^{(\infty)} \leq u_{i} \leq \bar{u}_{i}^{(\infty)}$ on $\bar{\Omega}$ for $i=1,2$.

Proof. See Pao [11, pp. 403-409].

It is easy to see that if all the data in (S), namely, $\partial \Omega, f_{i}, g_{i}, i=1,2$, are $C^{\infty}$-smooth, then the limits of monotone convergence $\underline{u}_{i}^{(\infty)}$ and $\bar{u}_{i}^{(\infty)}, i=1,2$, have regularity $C^{\infty}(\bar{\Omega})$ because the pairs $\left(\underline{u}_{1}^{(\infty)}, \underline{u}_{2}^{(\infty)}\right),\left(\bar{u}_{1}^{(\infty)}, \bar{u}_{2}^{(\infty)}\right),\left(\underline{u}_{1}^{(\infty)}, \bar{u}_{2}^{(\infty)}\right)$, or $\left(\bar{u}_{1}^{(\infty)}, \underline{u}_{2}^{(\infty)}\right)$ are classical solutions of $(\underline{S})$ for the respective quasimonotone types.

With the existence of an ordered subsolution and supersolution, by Theorem 2.1 any iterate $\left(\underline{u}_{1}^{(n)}, \underline{u}_{2}^{(n)}\right),\left(\underline{u}_{1}^{(n)}, \bar{u}_{2}^{(n)}\right),\left(\bar{u}_{1}^{(n)}, \underline{u}_{2}^{(n)}\right)$ and $\left(\bar{u}_{1}^{(n)}, \bar{u}_{2}^{(n)}\right)$ will lie in $\langle\underline{\mathbf{u}}, \overline{\mathbf{u}}\rangle$. Thus we may replace the product interval $J=\left[a_{1}, b_{1}\right] \times\left[a_{2}, b_{2}\right]$ with $\langle\underline{\mathbf{u}}, \overline{\mathbf{u}}\rangle$ throughout all of the preceding discussions.

We now need to introduce the following notations. Given any sufficiently smooth function $f\left(x, u_{1}, u_{2}\right)$ of three variables $\left(x, u_{1}, u_{2}\right)$, we define two associated functions $\mathcal{D}_{1} f$ and $\mathcal{D}_{2} f$ of five variables $\left(x, u_{1}, u_{2}, v_{1}, v_{2}\right)$ by

$$
\begin{aligned}
& \left(\mathcal{D}_{1} f\right)\left(x, u_{1}, u_{2}, v_{1}, v_{2}\right) \\
& \quad=\left\{\begin{aligned}
\frac{1}{2\left(u_{1}-v_{1}\right)}\left[f\left(x, u_{1}, u_{2}\right)-f\left(x, v_{1}, u_{2}\right)\right. & \\
\left.+f\left(x, u_{1}, v_{2}\right)-f\left(x, v_{1}, v_{2}\right)\right], & \text { if } u_{1} \neq v_{1}, \\
\frac{1}{2}\left[\frac{\partial f\left(x, u_{1}, u_{2}\right)}{\partial u_{1}}+\frac{\partial f\left(x, u_{1}, v_{2}\right)}{\partial u_{1}}\right], & \text { if } u_{1}=v_{1} ;
\end{aligned}\right.
\end{aligned}
$$




$$
\begin{aligned}
\left(\mathcal{D}_{2} f\right)\left(x, u_{1}, u_{2}, v_{1}, v_{2}\right) & \\
\quad= & \left\{\begin{array}{cc}
\frac{1}{2\left(u_{2}-v_{2}\right)}\left[f\left(x, u_{1}, u_{2}\right)-f\left(x, u_{1}, v_{2}\right)\right. \\
\left.+f\left(x, v_{1}, u_{2}\right)-f\left(x, v_{1}, v_{2}\right)\right], & \text { if } u_{2} \neq v_{2}, \\
\frac{1}{2}\left[\frac{\partial f\left(x, u_{1}, u_{2}\right)}{\partial u_{2}}+\frac{\partial f\left(x, v_{1}, v_{2}\right)}{\partial u_{2}}\right], & \text { if } u_{2}=v_{2} .
\end{array}\right.
\end{aligned}
$$

Then $\mathcal{D}_{1} f$ and $\mathcal{D}_{2} f$ are continuous functions of the five variables $\left(x, u_{1}, u_{2}, v_{1}, v_{2}\right)$, and we have

$$
\begin{aligned}
f\left(x, u_{1}, u_{2}\right)-f\left(x, v_{1}, v_{2}\right)= & \left(\mathcal{D}_{1} f\right)\left(x, u_{1}, u_{2}, v_{1}, v_{2}\right) \cdot\left(u_{1}-v_{1}\right) \\
& +\left(\mathcal{D}_{2} f\right)\left(x, u_{1}, u_{2}, v_{1}, v_{2}\right) \cdot\left(u_{2}-v_{2}\right) .
\end{aligned}
$$

Although convergence is guaranteed in Theorem 2.1, the rates are not known in general. However, in practical implementation, the success or failure of computation hinges crucially on the existence of a quantifiable rate of convergence - the faster, the more desirable. In order to obtain such explicit rates of convergence, as in [5], some additional assumption is required. We now formulate a condition that is sufficient to guarantee an algebraically fast rate of convergence. It is a generalization of assumption $[H]$ in our earlier study of the scalar semilinear elliptic equation. It is based upon estimations in $₫ 4$ for a $2 \times 2$ linear matrix problem in $\mathbb{R}^{2}$, but now generalized to an infinite dimensional setting. From the arguments that follow in $\S 4$, the reader may agree that the assumption is rather "generically tight":

$\begin{aligned} {\left[H_{2 \times 2}^{2}\right] \quad } & \min \left[\lambda_{1}^{*}+\mathcal{D}_{1} f_{1}\left(x, u_{1}, u_{2}, v_{1}, v_{2}\right)\right]>0, \\ & \min \left[\lambda_{2}^{*}+\mathcal{D}_{2} f_{2}\left(x, u_{1}, u_{2}, v_{1}, v_{2}\right)\right]>0,\end{aligned}$

for $x \in \bar{\Omega},\left(u_{1}, u_{2}\right) \in\langle\underline{\mathbf{u}}, \overline{\mathbf{u}}\rangle,\left(v_{1}, v_{2}\right) \in\langle\underline{\mathbf{u}}, \overline{\mathbf{u}}\rangle$.

Theorem 2.2. Let the semilinear system (S) be quasimonotone nonincreasing, quasimonotone nondecreasing, or mixed quasimonotone with pairs of ordered subsolutions $\left(\underline{u}_{1}, \underline{u}_{2}\right)$ and $\left(\bar{u}_{1}, \bar{u}_{2}\right)$. Let assumptions $\left[H_{2 \times 2}^{i}\right]$ hold for $i=1,2$, let the iteration be performed as in (2.15) -(2.20) for some $\bar{\lambda}=\left(\bar{\lambda}_{1}, \bar{\lambda}_{2}\right), \bar{\lambda}_{1} \geq 0, \bar{\lambda}_{2} \geq 0$, satisfying $\left[H_{2 \times 2}^{1}\right]$, and let

$$
u_{i}^{(n)} \equiv \underline{u}_{i}^{(n)} \quad\left(\text { resp. } u_{i}^{(n)} \equiv \bar{u}_{i}^{(n)}\right), \quad i=1,2 ; \quad n=0,1,2, \ldots,
$$

and

$$
u_{i}^{(\infty)} \equiv \underline{u}_{i}^{(\infty)} \quad\left(\text { resp. } u_{i}^{(\infty)} \equiv \bar{u}_{i}^{(\infty)}\right), \quad i=1,2,
$$

where $\underline{u}_{i}^{(\infty)}$ and $\bar{u}_{i}^{(\infty)}, i=1,2$, are promised by Theorem 2.1. Denote $e_{i}^{(m)}=$ $u_{i}^{(m)}-u_{i}^{(\infty)}, i=1,2$. Then there exist $a \bar{\lambda}$ and $a \theta_{\bar{\lambda}}, 0<\theta_{\bar{\lambda}}<1$, such that

$$
\left.\left\|\left(e_{1}^{(n)}, e_{2}^{(n)}\right)\right\|_{0, \Omega} \leq \theta_{\bar{\lambda}}^{n} \| e_{1}^{(0)}, e_{2}^{(0)}\right) \|_{0, \Omega}, \quad n=1,2, \ldots .
$$

Proof. Denote

$$
u^{(n)}(x)=\left(u_{1}^{(n)}(x), u_{2}^{(n)}(x)\right), \quad u^{(\infty)}(x)=\left(u_{1}^{(\infty)}(x), u_{2}^{(\infty)}(x)\right) .
$$

By Theorem 2.1, the $u_{i}^{(\infty)}, i=1,2$, satisfy

$$
\begin{cases}\bar{\lambda}_{i} u_{i}^{(\infty)}-\Delta u_{i}^{(\infty)}=F_{i, \bar{\lambda}_{i}}\left(\cdot, u^{(\infty)}\right), & i=1,2, \text { on } \Omega, \\ B_{i} u_{i}^{(\infty)}=g_{i}, & i=1,2, \text { on } \partial \Omega .\end{cases}
$$


Consider first the quasimonotone nonincreasing and quasimonotone nondecreasing cases. Then (2.15) holds with $\lambda_{i}=\bar{\lambda}_{i}$. Subtracting (2.27) from (2.15), we get

$$
\left\{\begin{array}{l}
\left(\bar{\lambda}_{i}-\Delta\right) e_{i}^{(n+1)}=\bar{\lambda}_{i} e_{i}^{(n)}-\left[f_{i}\left(\cdot, u^{(n)}\right)-f_{i}\left(\cdot, u^{(\infty)}\right)\right], \quad \text { on } \Omega, \\
B_{i} e_{i}^{(n+1)}=0, \quad \text { on } \partial \Omega, \\
i=1,2 .
\end{array}\right.
$$

Multiplying (2.28) by $e_{i}^{(n+1)}$ and integrating by parts, we obtain

$$
-\int_{\partial \Omega} \frac{\partial e_{i}^{(n+1)}}{\partial n} e_{i}^{(n+1)} d \sigma+\int_{\Omega}\left|\nabla e_{i}^{(n+1)}\right|^{2} d x+\bar{\lambda}_{i} \int_{\Omega}\left|e_{i}^{(n+1)}\right|^{2} d x=\int_{\Omega} H_{i}^{(n)} e_{i}^{(n+1)} d x,
$$

where

$$
H_{i}^{(n)} \equiv \bar{\lambda}_{i} e_{i}^{(n)}-\left[f_{i}\left(\cdot, u^{(n)}\right)-f_{i}\left(\cdot, u^{(\infty)}\right)\right] .
$$

Depending on whether $B_{i} e_{i}^{(n+1)}=0$ represents the homogeneous Dirichlet, Neumann or Robin boundary condition,

$$
e_{i}^{(n+1)} \equiv 0, \quad \frac{\partial}{\partial n} e_{i}^{(n+1)} \equiv 0, \quad \frac{\partial}{\partial n} e_{i}^{(n+1)}+\alpha_{i}(\cdot) e_{i}^{(n+1)} \equiv 0 \text { on } \partial \Omega,
$$

we have, respectively,

$$
\begin{aligned}
& \lambda_{i}^{*}=\inf _{v \in H_{0}^{1}(\Omega)} \frac{\int_{\Omega}|\nabla v|^{2} d x}{\|v\|_{0, \Omega}^{2}}, \quad \lambda_{i}^{*}=0, \\
& \lambda_{i}^{*}=\inf _{v \in H^{1}(\Omega)}\left[\int_{\partial \Omega} \alpha|v|^{2} d \sigma+\int_{\Omega}|\nabla v|^{2} d x\right] /\|v\|_{0, \Omega}^{2} .
\end{aligned}
$$

From (2.28)-(2.32), we obtain

$$
\left(\lambda_{i}^{*}+\bar{\lambda}_{i}\right) \int_{\Omega}\left|e_{i}^{(n+1)}\right|^{2} d x \leq \int_{\Omega} H_{i}^{(n)} e_{i}^{(n+1)} d x, \quad i=1,2 .
$$

By (2.25), we have

$$
H_{i}^{(n)}=\left[\bar{\lambda}_{i}-\mathcal{D}_{i} f_{i}\left(x, u^{(n)}(x), u^{(\infty)}(x)\right)\right] e_{i}^{(n)}(x)-\mathcal{D}_{\hat{i}} f_{i}\left(x, u^{(n)}(x), u^{(\infty)}(x)\right) e_{\hat{i}}^{(n)}(x),
$$

for

$$
(i, \hat{i})=(1,2),(2,1) .
$$

From (2.33) and (2.34), we get

$$
\left\{\begin{array}{l}
\int_{\Omega}\left|e_{i}^{(n+1)}\right|^{2} d x \leq \int_{\Omega}\left[\frac{\bar{\lambda}_{i}-\mathcal{D}_{i} f_{i}}{\bar{\lambda}_{i}+\lambda_{i}^{*}} e_{i}^{(n)} e_{i}^{(n+1)}-\frac{\mathcal{D}_{\hat{i}} f_{i}}{\bar{\lambda}_{i}+\lambda_{i}^{*}} e_{\hat{i}}^{(n)} e_{i}^{(n+1)}\right] d x \\
\text { for }(i, \hat{i}) \text { as in (2.35), and }\left(\mathcal{D}_{i} f_{i}, \mathcal{D}_{\hat{i}} f_{i}\right) \text { as in (2.34). }
\end{array}\right.
$$

Note that the above step is analogous to (4.12) and (4.13) given below in $\$ 4$, but here we have an infinite dimensional setting. We may now rewrite (2.36) in matrix 
form:

$$
\int_{\Omega}\left|e^{(n+1)}\right|^{2} d x \leq \int_{\Omega}\left[e_{1}^{(n+1)} e_{2}^{(n+1)}\right] \mathbb{A}\left[\begin{array}{c}
e_{1}^{(n)} \\
e_{2}^{(n)}
\end{array}\right] d x
$$

where

$$
\begin{aligned}
\mathbb{A} & =\mathbb{A}\left(\bar{\lambda}_{1}, \bar{\lambda}_{2}, x, u^{(n)}(x), u^{(\infty)}(x)\right] \\
& \equiv\left[\begin{array}{cc}
\frac{\bar{\lambda}_{1}-\mathcal{D}_{1} f_{1}\left(x, u^{(n)}(x), u^{(\infty)}(x)\right)}{\lambda_{1}+\lambda_{1}^{*}} & -\frac{\mathcal{D}_{2} f_{1}\left(x, u^{(n)}(x), u^{(\infty)}(x)\right)}{\lambda_{1}+\lambda_{1}^{*}} \\
-\frac{\mathcal{D}_{1} f_{2}\left(x, u^{(n)}(x), u^{(\infty)}(x)\right)}{\lambda_{2}+\lambda_{2}^{*}} & \frac{\bar{\lambda}_{2}-\mathcal{D}_{2} f_{2}\left(x, u^{(n)}(x), u^{(\infty)}(x)\right)}{\lambda_{2}+\lambda_{2}^{*}}
\end{array}\right] .
\end{aligned}
$$

We can now apply Theorem 4.2 and (4.12)-(4.14) in $\$ 4$ to get (cf. (4.2) for the norm of a $2 \times 2$ matrix in $\mathbb{R}^{2}$ )

$\|\mathbb{A}(\cdot)\|_{\mathbb{R}^{2}}$

$$
\begin{aligned}
\leq \frac{1}{2}\{ & {\left[\left(\frac{\bar{\lambda}_{1}-\mathcal{D}_{1} f_{1}(\cdot)}{\bar{\lambda}_{1}+\lambda_{1}^{*}}+\frac{\bar{\lambda}_{2}-\mathcal{D}_{2} f_{2}(\cdot)}{\bar{\lambda}_{2}+\lambda_{2}^{*}}\right)^{2}+\left(-\frac{\mathcal{D}_{2} f_{1}(\cdot)}{\bar{\lambda}_{1}+\lambda_{1}^{*}}+\frac{\mathcal{D}_{1} f_{2}(\cdot)}{\bar{\lambda}_{2}+\lambda_{2}^{*}}\right)^{2}\right]^{1 / 2} } \\
& \left.+\left[\left(\frac{\bar{\lambda}_{1}-\mathcal{D}_{1} f_{1}(\cdot)}{\bar{\lambda}_{1}+\lambda_{1}^{*}}-\frac{\bar{\lambda}_{2}-\mathcal{D}_{2} f_{2}(\cdot)}{\bar{\lambda}_{2}+\lambda_{2}^{*}}\right)^{2}+\left(\frac{\mathcal{D}_{2} f_{1}(\cdot)}{\bar{\lambda}_{1}+\lambda_{1}^{*}}-\frac{\mathcal{D}_{1} f_{2}(\cdot)}{\bar{\lambda}_{2}+\lambda_{2}^{*}}\right)^{2}\right]^{1 / 2}\right\} .
\end{aligned}
$$

Note that (2.38) is exactly in the same form as (4.14) under the correspondence

$$
\lambda_{1} \leftrightarrow \bar{\lambda}_{1}, \lambda_{2} \leftrightarrow \bar{\lambda}_{2}, a \leftrightarrow \mathcal{D}_{1} f_{1}(\cdot), b \leftrightarrow \mathcal{D}_{1} f_{2}(\cdot), c \leftrightarrow \mathcal{D}_{2} f_{1}(\cdot), d \leftrightarrow \mathcal{D}_{2} f_{2}(\cdot) .
$$

Therefore, by applying Theorem 4.3 , we see that by $\left[H_{2 \times 2}^{2}\right]$ we can find some $\bar{\lambda}_{1}>0, \bar{\lambda}_{2}>0$, sufficiently large, such that

$$
\left\|\mathbb{A}\left(\bar{\lambda}_{1}, \bar{\lambda}_{2}, x, u_{1}, u_{2}, v_{1}, v_{2}\right)\right\| \leq \theta_{\bar{\lambda}}<1, \quad \forall x \in \bar{\Omega}, \forall\left(u_{1}, u_{2}\right),\left(v_{1}, v_{2}\right) \in\langle\underline{\mathbf{u}}, \overline{\mathbf{u}}\rangle .
$$

From (2.37), we get

$$
\int_{\Omega}\left|e^{(n+1)}\right|^{2} d x \leq \theta_{\bar{\lambda}}\left\|e^{(n+1)}\right\|_{0, \Omega}\left\|e^{(n)}\right\|_{0, \Omega},
$$

and so

$$
\left\|e^{(n+1)}\right\|_{0, \Omega} \leq \theta_{\bar{\lambda}}\left\|e^{(n)}\right\|_{0, \Omega}, \quad n=0,1,2, \ldots .
$$

Therefore (2.26) is obtained.

By the intermediate value theorem and the continuity of $\partial f_{i} / \partial u_{i}, i=1,2$, we easily see from (2.23) that $\left[\mathrm{H}_{2 \times 2}^{2}\right]$ is equivalent to

$$
\begin{aligned}
{\left[H_{2 \times 2}^{2}\right]: } & \min \left[\lambda_{1}^{*}+\frac{\partial f_{1}\left(x, u_{1}, u_{2}\right)}{\partial u_{1}}\right]>0, \quad \min \left[\lambda_{2}^{*}+\frac{\partial f_{2}\left(x, u_{1}, u_{2}\right)}{\partial u_{2}}\right]>0, \\
& \text { for } x \in \bar{\Omega},\left(u_{1}, u_{2}\right) \in\langle\underline{\mathbf{u}}-\delta, \overline{\mathbf{u}}+\delta\rangle, \text { for some small } \delta>0 .
\end{aligned}
$$

By Remark 4.6] in $\S 4$, we see that, generically, $\left[\mathrm{H}_{2 \times 2}^{2}\right]$ is about the tightest we can get in order for (2.26) to hold for some $\theta_{\bar{\lambda}}, 0<\theta_{\bar{\lambda}}<1$. 


\section{ERror anALYSis FOR a GALERKIN BOUNDARY ELEMENT MONOTONE ITERATION SCHEME}

We now introduce a Galerkin boundary element scheme for computing (2.15), (2.19) or (2.20), and analyze the errors. Let $E(x-y ; \lambda)$ be the fundamental solution of the Helmholtz operator $\Delta-\lambda$ satisfying

$$
(\Delta-\lambda) E(x-y ; \lambda)=-\delta(x-y), \quad \lambda>0, \quad x, y \in \mathbb{R}^{N} .
$$

As in Part I [5], denote the volume potential by $\widetilde{V}_{\lambda}$ :

$$
\left(\widetilde{V}_{\lambda} \phi\right)(x)=\int_{\Omega} E(x-y ; \lambda) \phi(y) d y, \quad x \in \Omega,
$$

and the simple-layer potential by $\widetilde{S}_{\lambda}$ :

$$
\left(\widetilde{S}_{\lambda} \eta\right)(x)=\int_{\partial \Omega} E(x-y ; \lambda) \eta(y) d \sigma_{y}, \quad x \in \bar{\Omega} .
$$

Also, let $S_{\lambda}$ and $V_{\lambda}$ be, respectively, the trace of $\widetilde{S}_{\lambda}$ and $\widetilde{V}_{\lambda}$ :

$$
\left.S_{\lambda} \eta \equiv\left(\widetilde{S}_{\lambda} \eta\right)\right|_{\partial \Omega},\left.\quad V_{\lambda} \phi \equiv\left(V_{\lambda} \phi\right)\right|_{\partial \Omega} .
$$

The normal derivative of the volume potential is denoted by

$$
\left(V_{\lambda}^{\prime} \phi\right)(x)=\int_{\Omega} \frac{\partial E(x-y ; \lambda)}{\partial n_{x}} \phi(y) d y, \quad x \in \partial \Omega .
$$

In addition, we define the boundary operator $K_{\lambda}$ :

$$
\left(K_{\lambda} \eta\right)(x)=\int_{\partial \Omega} \frac{\partial E(x-y ; \lambda)}{\partial n_{x}} \eta(y) d \sigma_{y}, \quad x \in \partial \Omega .
$$

The regularity properties of these integral operators are summarized below.

Theorem 3.1. Let $\lambda>0$. Then

(i) $\widetilde{V}_{\lambda}$ maps continuously from $H^{s}(\Omega)$ into $H^{s+2}(\Omega), \forall s \geq-1$;

(ii) $V_{\lambda}$ maps continuously from $H^{s}(\Omega)$ into $H^{s+\frac{3}{2}}(\partial \Omega), \forall s \geq-1$;

(iii) $V_{\lambda}^{\prime}$ maps continuously from $H^{s}(\Omega)$ into $H^{s+\frac{1}{2}}(\partial \Omega), \forall s \geq-1$;

(iv) $\widetilde{S}_{\lambda}$ maps continuously from $H^{s}(\partial \Omega)$ into $H^{s+\frac{3}{2}}(\Omega), \forall s \in \mathbb{R}$;

(v) $S_{\lambda}$ maps isomorphically from $H^{s}(\partial \Omega)$ onto $H^{s+1}(\partial \Omega)$, and is positive definite, $\forall s \in \mathbb{R} ; S_{\lambda}$ is a strongly elliptic pseudodifferential operator of order -1 ;

(vi) $K_{\lambda}$ maps continuously from $H^{s}(\partial \Omega)$ into

$$
\begin{cases}H^{s+1}(\partial \Omega), \forall s \in \mathbb{R}, & \text { if } N \geq 3, \\ C^{\infty}(\partial \Omega), \forall s \in \mathbb{R}, & \text { if } N=2 .\end{cases}
$$

Furthermore, $\frac{1}{2} I+K_{\lambda}$ maps $H^{s}(\partial \Omega)$ onto $H^{s}(\partial \Omega)$ isomorphically for every $s \in \mathbb{R}$ such that $\frac{1}{2} I+K_{\lambda}=\left.\left(\frac{\partial}{\partial n} \widetilde{S}_{\lambda}\right)\right|_{\partial \Omega}$, and $\frac{1}{2} I+K_{\lambda}$ is a strongly elliptic pseudodifferential operator of order 0 .

(vii) For $\alpha(\cdot)$ given as in (2.4), the operator

$$
T_{\lambda} \equiv \frac{1}{2} I+K_{\lambda}+\alpha(\cdot) S_{\lambda}
$$

is a strongly elliptic pseudodifferential operator of order 0 mapping isomorphically from $H^{s}(\partial \Omega)$ onto $H^{s}(\partial \Omega) \forall s \in \mathbb{R}$. 
Return to the iteration (2.15)-(2.20). In the two boundary conditions $B_{i} u_{i}=g_{i}$ for $i=1,2$, we have several different possible combinations for $\left(B_{1}, B_{2}\right)$, say, for example, (Dirichlet, Dirichlet), (Dirichlet, Neumann) or (Dirichlet, Robin) type. In the subsequent error analysis, the arguments for the Neumann type boundary condition are identical to those for the Robin type. Therefore, for definiteness, let us treat the most representative case, $\left(B_{1}, B_{2}\right)=$ (Dirichlet, Robin), i.e.,

$$
\left.\left.B_{1} u_{1} \equiv u_{1}\right|_{\partial \Omega}=g_{1} ; \quad B_{2} u_{2}=\left.\left(\frac{\partial u_{2}}{\partial n}+\alpha(\cdot) u\right)\right|_{\partial \Omega}=g_{2} \quad \text { (cf. (2.4) }\right) .
$$

Noting from Part I [5] that the indirect formulation is more advantageous, we continue to adopt it here by using the ansatz [5. (3.8)], that the solution is a sum of a simple-layer potential plus a volume (Newtonian) potential (which is often called a domain integral):

$$
\begin{aligned}
u_{i}^{(n+1)}(x)= & \left(\widetilde{S}_{\lambda_{i}} \eta_{i}^{(n+1)}\right)(x)-\left(\widetilde{V}_{\lambda_{i}} F_{i, \lambda_{i}}\left(\cdot, u_{1}^{(n)}, u_{2}^{(n)}\right)\right)(x) \\
= & \int_{\partial \Omega} E\left(x-y ; \lambda_{i}\right) \eta_{i}^{(n+1)}(y) d \sigma_{y} \\
& -\int_{\Omega} E\left(x-y ; \lambda_{i}\right) F_{i, \lambda_{i}}\left(y, u_{1}^{(n)}(y), u_{2}^{(n)}(y)\right) d y, \\
& x \in \bar{\Omega}, \quad i=1,2,
\end{aligned}
$$

where $\eta_{i}^{(n+1)}, i=1,2$, are the unknown simple layer densities to be solved, on $\partial \Omega$ only. Applying the boundary conditions in (2.2) to (3.3), we get the following two boundary integral equations for the determination of $\eta_{i}^{(n+1)}, i=1,2$ :

$$
\left.\begin{array}{c}
S_{\lambda_{1}} \eta_{1}^{(n+1)}=g_{1}+V_{\lambda_{1}} F_{1, \lambda_{1}}^{(n)}, \\
T_{\lambda_{2}} \eta_{2}^{(n+1)}=g_{2}+\left(V_{\lambda_{2}}^{\prime}+\alpha V_{\lambda_{2}}\right) F_{2, \lambda_{2}}^{(n)}
\end{array}\right\} \text { on } \partial \Omega,
$$

where

$$
\left.F_{i, \lambda_{i}}^{(n)} \equiv F_{i, \lambda_{i}}\left(\cdot, u_{1}^{(n)}, u_{2}^{(n)}\right)\right) .
$$

Lemma 3.2. Let $g_{i} \in C^{\infty}(\partial \Omega)$ and $F_{i, \lambda_{i}}^{(n)} \in C^{\infty}(\bar{\Omega})$ for $i=1,2$ in (2.2) and (3.6). Then there exist unique $\eta_{1}^{(n+1)}, \eta_{2}^{(n+1)} \in C^{\infty}(\partial \Omega)$ which are, respectively, the solution of the BIEs (3.4) and 3.5.

Proof. The BIE (3.4) is solvable with a unique solution $\eta_{1}^{(n+1)}$ because the property (v) in Theorem 3.1 .

The BIE (3.5) is also solvable with a unique solution $\eta_{2}^{(n+1)}$ because of property (vii) in Theorem 3.1 .

The following theorem is a generalization and combination of 5, Theorem 3.1 and Corollary 3.3]. The indicated antimonotone property is useful and has also been confirmed numerically.

Theorem 3.3. Let the assumptions of Theorem [2.1 hold, including [ $\left.H_{2 \times 2}^{1}\right]$ but not $\left[H_{2 \times 2}^{2}\right]$, Let the boundary conditions be given as $(\underline{3.2})$, and let $\underline{\eta}_{1}^{(n+1)}, \bar{\eta}_{1}^{(n+1)}$, $\underline{\eta}_{2}^{(n+1)}, \bar{\eta}_{2}^{(n+1)}$ be the simple layer densities in (3.4) and (3.5) corresponding, respectively, to $\underline{u}_{1}^{(n+1)}, \bar{u}_{1}^{(n+1)}, \underline{u}_{2}^{(n+1)}, \bar{u}_{2}^{(n+2)}$ in the quasimonotone iteration. Then 
we have the antimonotone property

$$
\underline{\eta}_{i}^{(n)} \geq \underline{\eta}_{i}^{(n+1)} \geq \bar{\eta}_{i}^{(n+1)} \geq \bar{\eta}_{i}^{(n)} \quad \text { on } \partial \Omega, \quad i=1,2, n=0,1,2, \ldots
$$

Consequently, $\underline{\eta}_{i}^{(n)}$ and $\bar{\eta}_{i}^{(n)}$, converge monotonically and respectively, to some $\underline{\eta}_{i}^{(\infty)}$ and $\bar{\eta}_{i}^{(\infty)}$, naturally corresponding to $\underline{u}_{i}^{(\infty)}, \bar{u}_{i}^{(\infty)}$, respectively, for $i=1,2$ in Theorem 2.1.

Proof. The boundary operator $S_{\lambda_{1}}$ in (3.4) is the same simple-layer boundary operator as in [5, (3.12)], so we can apply [5, Theorem 3.1] directly. The boundary operator $T_{\lambda_{2}}$ in (3.5) corresponds to the Robin boundary $B_{2}$ in (3.2), and therefore, we need to establish an analogue of [5] Theorem 3.1] for the operator $T_{\lambda_{2}}$ based upon the representation (3.3). Let us go to the proof of [5, Theorem 3.1, pp. 951955]; we need only modify certain segments therein. First, inherit all the work there, except that in [5, $(3.25)_{2}$ and $\left.(3.26)_{2}\right]$, we change the homogeneous Dirichlet boundary conditions to the homogeneous Robin conditions. But note that [5]. $(3.33)_{2}$ ] remains unchanged. Then the rest of all the arguments in [5] pp. 954-955] remain the same, except that at [5, line 13, p. 955], we now write instead

$$
\begin{aligned}
\frac{\partial}{\partial n}\left[w^{+}\right. & \left.(x)-w^{-}(x)\right]+\alpha(x)\left[w^{+}(x)-w^{-}(x)\right]=\frac{\partial}{\partial n}\left[w^{+}(x)-w^{-}(x)\right] \\
& =\cdots \cdots(\text { same as in }[5, \text { lines } 14-18, \text { p. 955]) } \\
& =\eta_{1}(x)-\eta_{2}(x) \geq 0 .
\end{aligned}
$$

Therefore the proof is complete.

Throughout the discussions in the rest of this section, without further mention, we have assumed $\left[H_{2 \times 2}^{i}\right], i=1,2$.

For later use, we define, for given $\lambda=\left(\lambda_{1}, \lambda_{2}\right)$,

$$
\begin{aligned}
M_{\lambda}= & \max \left\{\left[\left(\lambda_{1}-\mathcal{D}_{1} f_{1}\left(x, u_{1}, u_{2}, v_{1}, v_{2}\right)\right)^{2}+\left(\mathcal{D}_{2} f_{1}\left(x, u_{1}, u_{2}, v_{1}, v_{2}\right)^{2}\right]^{1 / 2},\right.\right. \\
& {\left.\left[\left(\lambda_{2}-\mathcal{D}_{2} f_{2}\left(x, u_{1}, u_{2}, v_{1}, v_{2}\right)\right)^{2}+\left(\mathcal{D}_{1} f_{2}\left(x, u_{1}, u_{2}, v_{1}, v_{2}\right)\right)^{2}\right]^{1 / 2}\right\} } \\
& x \in \bar{\Omega} ;\left(u_{1}, u_{2}\right),\left(v_{1}, v_{2}\right) \in\langle\underline{\mathbf{u}}-\delta, \overline{\mathbf{u}}+\delta\rangle .
\end{aligned}
$$

As in Part I [5, let $\mathcal{S}_{h}, 0<h \leq h_{0}$, be a 1-parameter family of finite-dimensional boundary element approximation spaces that form an $(\ell, m)$-system $\mathcal{S}_{h}^{\ell, m}$, with $\ell$, $m \in \mathbb{Z}^{+} \equiv\{0,1,2, \ldots\}, \ell \geq m+1$, on $\partial \Omega$, in the sense of Babuška and Aziz [3]. The Galerkin boundary element scheme to solve (3.4) and (3.5) is:

(i) Initialization: Set $u_{i, h}^{(0)}(x)=\underline{u}_{i}(x)$ or $\bar{u}_{i}(x), i=1,2$, according to whether the semilinear system is quasimonotone nonincreasing, nondecreasing, or mixed, and according to (2.15)-(2.20).

(ii) Choose $\lambda_{i} \geq 0, i=1,2$, satisfying $\left[H_{2 \times 2}^{1}\right]$ and $\left[H_{2 \times 2}^{2}\right]$.

(iii) For each $n=0,1,2, \ldots$, and $h, 0<h \leq h_{0}$, find $\eta_{i, h}^{(n+1)} \in \mathcal{S}_{h}$ such that

$$
\left.\begin{array}{l}
\left\langle S_{\lambda_{1}} \eta_{1, h}^{(n+1)}, \phi_{h}\right\rangle=\left\langle g_{1}+V_{\lambda_{1}} F_{1, \lambda_{1}, h}^{(n)}, \phi_{h}\right\rangle \\
\left\langle T_{\lambda_{2}} \eta_{2, h}^{(n+1)}, \phi_{h}\right\rangle=\left\langle g_{2}+\left(V_{\lambda_{2}}^{\prime}+\alpha V_{\lambda_{2}}\right) F_{2, \lambda_{2}, h}^{(n)}, \phi_{h}\right\rangle
\end{array}\right\} \quad \forall \phi_{h} \in \mathcal{S}_{h},
$$

where $\langle$,$\rangle signifies the L^{2}(\partial \Omega)$ inner product, and

$$
F_{i, \lambda_{i}, h}^{(n)} \equiv F_{i, \lambda_{i}}\left(\cdot, u_{1, h}^{(n)}, u_{2, h}^{(n)}\right) .
$$


(iv) Define

$$
u_{i, h}^{(n+1)}(x)=\left(\widetilde{S}_{\lambda_{i}} \eta_{i, h}^{(n+1)}\right)(x)-\left(\widetilde{V}_{\lambda_{i}} F_{i, \lambda_{i}, h}^{(n)}\right)(x), \quad x \in \bar{\Omega}, n=0,1,2, \ldots .
$$

Note that the two equations in (3.8) can be computed separately. Let us state the main theorem of error estimates.

Theorem 3.4. Let $\left\{\mathcal{S}_{h} \mid 0<h \leq h_{0}\right\}$ be a family of $(\ell, m)$-systems on $\partial \Omega$ with $\ell \geq 2, m \geq 1$, and let $N=2$ or 3 . Assume $\left[H_{2 \times 2}^{i}\right], i=1,2$. Then there exist $K_{1}>0, K_{2}>0$ (the same as in (E4) and (E5) below), $\rho_{\lambda}, 0<\rho_{\lambda}<1$, and a small $\bar{h}_{0}>0$ satisfying 3.21 such that for any $r, 0 \leq r<2$, we have

$$
\begin{aligned}
& \left\|\left(u_{1, h}^{(n)}-u_{1}^{(\infty)}, u_{2, h}^{(n)}-u_{2}^{(\infty)}\right)\right\|_{r, \Omega} \\
& \quad \leq K_{1} h^{2-r}\left\|\left(u_{1}^{(\infty)}, u_{2}^{(\infty)}\right)\right\|_{2, \Omega}+K_{2} \rho_{\lambda}^{n}\left\|\left(e_{1}^{(0)}, e_{2}^{(0)}\right)\right\|_{0, \Omega},
\end{aligned}
$$

for all $n=1,2, \ldots, 0<h \leq \bar{h}_{0}$. Therefore $\left(u_{1, h}^{(n)}, u_{2, h}^{(n)}\right)$ converges to $\left(u_{1}^{(\infty)}, u_{2}^{(\infty)}\right)$ as $n \rightarrow \infty$ and $h \downarrow 0$. Also, for $-\frac{3}{2} \leq s<\frac{1}{2}$, there are constants $K_{3, s}>0$ and $K_{4, s}>0$ such that

$$
\begin{aligned}
& \left\|\left(\eta_{1, h}^{(n)}-\eta_{1}^{(\infty)}, \eta_{2, h}^{(n)}-\eta_{2}^{(\infty)}\right)\right\|_{s, \partial \Omega} \\
& \quad \leq K_{3, s} h^{\frac{1}{2}-s}\left\|\left(u_{1}^{(\infty)}, u_{2}^{(\infty)}\right)\right\|_{2, \Omega}+K_{4, s} \rho_{\lambda}^{n}\left\|\left(e_{1}^{(0)}, e_{2}^{(0)}\right)\right\|_{2, \Omega},
\end{aligned}
$$

for all $n=1,2, \ldots, 0<h \leq \bar{h}_{0}$.

In particular, if $N=2$ and if $\left\{\mathcal{S}_{h} \mid 0<h \leq h_{0}\right\}$ is a family of $S_{h}^{d}$ spaces of smoothest splines of degree $d \geq 0$ with respect to a quasiuniform mesh on $\partial \Omega$, then (3.10) holds for $0 \leq r<2$, and (3.11) holds for $-(d+2) \leq s<1 / 2$.

Proof. See (E1)-(E6) below and [5, Theorems 4.10 and 4.12].

We will be concise in deriving error estimates, since much of the procedure is similar to the work in [5]. The estimation is done through a string of comparisons. Note that

(I) The exact simple-layer densities $\left(\eta_{1}^{(n+1)}, \eta_{2}^{(n+1)}\right)$ for the iterative solution $\left(u_{1}^{(n+1)}, u_{2}^{(n+1)}\right)$ (cf. (2.15) ) satisfy the BIEs (3.4) and (3.5).

(II) The Galerkin boundary element approximation

$$
\left(\eta_{1, h}^{(n+1)}, \eta_{2, h}^{(n+1)}\right)
$$

for $\left(\eta_{1}^{(n+1)}, \eta_{2}^{(n+1)}\right)$ satisfies (3.8) .

(III) The numerical solution

$$
\left(u_{1, h}^{(n+1)}, u_{2, h}^{(n+1)}\right)
$$

approximating $\left(u_{1}^{(n+1)}, u_{2}^{(n+1)}\right)$ at each step of the iteration is defined from $\left(\eta_{1, h}^{(n+1)}, \eta_{2, h}^{(n+1)}\right)$ through $(\underline{3.9})$. The errors are defined to be

$$
e_{i, h}^{(n+1)}=u_{i, h}^{(n+1)}-u_{i}^{(n+1)}, \quad i=1,2 ; \quad n=0,1,2, \ldots ;
$$

(IV) An intermediate simple-layer density pair $\left(\zeta_{1, h}^{(n+1)}, \zeta_{2, h}^{(n+1)}\right)$ on $\partial \Omega$ for the comparison purpose is defined to be the unique solutions of the BIEs

$$
\left\{\begin{array}{l}
S_{\lambda_{1}} \zeta_{1, h}^{(n+1)}=g_{1}-V_{\lambda_{1}} F_{1, \lambda_{1}, h}^{(n)}, \\
T_{\lambda_{2}} \zeta_{2, h}^{(n+1)}=g_{2}-\left(V_{\lambda_{2}}^{\prime}+\alpha V_{\lambda_{2}}\right) F_{2, \lambda_{2}, h}^{(n)},
\end{array} \quad \text { on } \partial \Omega, \quad n=0,1,2, \ldots,\right.
$$

(as guaranteed in the same way by Lemma [3.2); 
(V) An intermediate solution pair $\left(w_{1, h}^{(n+1)}, w_{2, h}^{(n+1)}\right)$ on $\bar{\Omega}$ for the comparison purpose is defined by

$$
\begin{aligned}
w_{i, h}^{(n+1)}(x)=\left(\widetilde{S}_{\lambda_{i}} \zeta_{i, h}^{(n+1)}\right)(x)-\left(\widetilde{V}_{\lambda_{i}} F_{i, \lambda_{i}, h}^{(n)}\right)(x), & \\
x & \in \bar{\Omega}, \quad i=1,2 ; \quad n=0,1,2, \ldots
\end{aligned}
$$

It is obvious that $w_{i, h}^{(n+1)}, i=1,2$, satisfy

$$
\begin{cases}\left(\lambda_{i}-\Delta\right) w_{i, h}^{(n+1)}=F_{i, \lambda_{i}, h}^{(n)} & \text { on } \Omega \\ B_{i} w_{i, h}^{(n+1)}=g_{i} & \text { on } \partial \Omega \\ i=1,2 ; n=0,1,2, \ldots & \end{cases}
$$

(VI) The limit of $\left(u_{1}^{(n+1)}, u_{2}^{(n+1)}\right)$ as $n \rightarrow \infty$ is $\left(u_{1}^{(\infty)}, u_{2}^{(\infty)}\right)$, as guaranteed by Theorem 2.1. Note that $\left(u_{1}^{\infty}, u_{2}^{\infty}\right)=\left(\underline{u}_{1}^{(\infty)}, \underline{u}_{2}^{(\infty)}\right)$, or $\left(\bar{u}_{1}^{(\infty)}, \bar{u}_{2}^{(\infty)}\right)$, or $\left(\underline{u}_{1}^{(\infty)}, \bar{u}_{2}^{(\infty)}\right)$, or $\left(\bar{u}_{1}^{(\infty)}, \underline{u}_{2}^{(\infty)}\right)$ according to Theorem 2.1. The pair $\left(u_{1}^{(\infty)}, u_{2}^{(\infty)}\right)$ is an exact solution of $(\mathbb{S})$. Corresponding to $\left(u_{1}^{(\infty)}, u_{2}^{(\infty)}\right)$, we have simplelayer densities $\left(\eta_{1}^{(\infty)}, \eta_{2}^{(\infty)}\right)$ which are solutions of the BIEs

$$
\left\{\begin{array}{l}
S_{\lambda_{1}} \eta_{1}^{(\infty)}=g_{1}+V_{\lambda_{1}} F_{1, \lambda_{1}}\left(\cdot, u_{1}^{(\infty)}, u_{2}^{(\infty)}\right) \\
T_{\lambda_{2}} \eta_{2}^{(\infty)}=g_{2}+\left(V_{\lambda_{2}}^{\prime}+\alpha V_{\lambda_{2}}\right) F_{2, \lambda_{2}}\left(\cdot, u_{1}^{(\infty)}, u_{2}^{(\infty)}\right)
\end{array} \quad \text { on } \partial \Omega .\right.
$$

The initial error $\left(e_{1}^{(0)}, e_{2}^{(0)}\right)$ is defined to be

$$
e_{i}^{(0)}=u_{i}^{(0)}-u_{i}^{(\infty)}
$$

The glossary of error estimates can now be stated sequentially as follows:

$$
\begin{aligned}
\left\|\eta_{i, h}^{(n+1)}-\zeta_{i, h}^{(n+1)}\right\|_{t, \partial \Omega} \leq C_{i, s, t} h^{t-s}\left\|\zeta_{i, h}^{(n+1)}\right\|_{s, \partial \Omega}, \\
i=1,2 ; \quad n=0,1,2, \ldots,
\end{aligned}
$$

where $C_{i, s, t}, i=1,2$, are positive constants independent of $n$ and $h$, and

$$
2 \omega-\ell \leq t \leq s \leq \ell, \quad t \leq m, \quad-\ell \leq \omega \leq m, \quad-m \leq \omega \leq t,
$$

with

$$
\omega \equiv\left\{\begin{array}{cc}
-\frac{1}{2}, & i=1 \\
0, & i=2 .
\end{array}\right.
$$

Proof. Cf. [5, Lemma 4.2 and §6], Hsiao and Wendland [6, Cor. 2.1] and Ruotsalainen and Saranen [12, Cor. 3].

(E2) Let $N=2$ or 3 , and

$$
\varepsilon_{0}(N)= \begin{cases}0, & N=2, \\ \text { an arbitrarily small positive number, } 0<\varepsilon_{0}(3)<1 / 2, & N=3 .\end{cases}
$$


Then for $\ell \geq 2, m \geq 1$, and some sufficiently small $h_{0}$, there are positive constants $K_{0}$ and $K_{\varepsilon_{0}(N)}$ such that

$$
\begin{aligned}
\| \eta_{i, h}^{(n+1)}- & \zeta_{i, h}^{(n+1)} \|_{\varepsilon_{0}(N), \partial \Omega} \\
\leq & K_{\varepsilon_{0}(N)} h^{\frac{1}{2}-\varepsilon_{0}(N)} \cdot\left[\left\|u_{i}^{(\infty)}\right\|_{2, \Omega}+M_{\lambda} \theta_{\lambda}^{n}\left\|\left(e_{1}^{(0)}, e_{2}^{(0)}\right)\right\|_{0, \Omega}\right. \\
& \left.+\left\|F_{i, \lambda_{i}, h}^{(n)}-F_{i, \lambda_{i}}^{(n)}\right\|_{0, \Omega}\right], \\
\| \eta_{i, h}^{(n+1)}- & \zeta_{i, h}^{(n+1)} \|_{-\frac{3}{2}, \partial \Omega} \\
\leq & K_{0} h^{2}\left[\left\|u_{i}^{(\infty)}\right\|_{2, \Omega}+M_{\lambda} \theta_{\lambda}^{n}\left\|\left(e_{1}^{(0)}, e_{2}^{(0)}\right)\right\|_{0, \Omega}\right. \\
& \left.+\left\|F_{i, \lambda_{i}, h}^{(n)}-F_{i, \lambda_{i}}^{(n)}\right\|_{0, \Omega}\right],
\end{aligned}
$$

for $i=1,2, n=0,1,2, \ldots, 0<h \leq h_{0}$.

Proof. Cf. [5, Lemma 4.3 and $\S 6]$.

(E3) Let $N=2$ or 3. For $\varepsilon_{0}(N)$ given by (3.17) and for $\beta$ with

$$
\begin{cases}0<\beta<1 / 2, & \text { if } N=2, \\ \beta=\varepsilon_{0}(3), & \text { if } N=3,\end{cases}
$$

there exist positive constants $K_{0}^{\prime}$ and $K_{\varepsilon_{0}(N)}^{\prime}$ such that

$$
\begin{aligned}
\left\|u_{i, h}^{(n+1)}-w_{i, h}^{(n+1)}\right\|_{C^{0, \beta}(\bar{\Omega}) \leq} \leq & K_{\varepsilon_{0}(N)}^{\prime} h^{\frac{1}{2}-\varepsilon_{0}(N)}\left[\left\|u_{i}^{(\infty)}\right\|_{2, \Omega}+M_{\lambda} \theta_{\lambda}^{n}\left\|\left(e_{1}^{(0)}, e_{2}^{(0)}\right)\right\|_{0, \Omega}\right. \\
& \left.+\left\|F_{i, \lambda_{i}, h}^{(n)}-F_{i, \lambda_{i}}^{(n)}\right\|_{0, \Omega}\right] \\
\left\|u_{i, h}^{(n+1)}-w_{i, h}^{(n+1)}\right\|_{0, \Omega} \leq & K_{0}^{\prime} h^{2}\left[\left\|u_{i}^{(\infty)}\right\|_{2, \Omega}+M_{\lambda} \theta_{\lambda}^{n}\left\|\left(e_{1}^{(0)}, e_{2}^{(0)}\right)\right\|_{0, \Omega}\right. \\
& \left.+\left\|F_{i, \lambda_{i}, h}^{(n)}-F_{i, \lambda_{i}}^{(n)}\right\|_{0, \Omega}\right],
\end{aligned}
$$

for $i=1,2, n=0,1,2, \ldots, 0<h \leq h_{0}$, where $K_{\varepsilon_{0}(N)}^{\prime}$ may grow unbounded as $\varepsilon_{0}(3) \downarrow 0$ when $N=3$.

Proof. Cf. 5, Lemma 4.5].

(E4) Let $N=2$ or 3 , and let $\beta$ satisfy (3.19). Then

$$
\begin{aligned}
\left\|w_{i, h}^{(n+1)}-u_{i}^{(n+1)}\right\|_{C^{0, \beta}(\bar{\Omega})} & \leq K_{1}\left\|F_{i, \lambda_{i}, h}^{(n)}-F_{i, \lambda_{i}}^{(n)}\right\|_{0, \Omega}, \\
\left\|w_{i, h}^{(n+1)}-u_{i}^{(n+1)}\right\|_{0, \Omega} & \leq \frac{1}{\lambda_{i}+\lambda_{i}^{*}}\left\|F_{i, \lambda_{i}, h}^{(n)}-F_{i, \lambda_{i}}^{(n)}\right\|_{0, \Omega}
\end{aligned}
$$

for some $K_{1}>0$ independent of $n, h$ and $\beta$, and for $i=1,2, n=0,1,2, \ldots, 0<$ $h \leq h_{0}$.

Proof. Cf. [5, Lemmas 4.6 and 4.7]. 
(E5) Let $N=2$ or 3 , and let $\beta$ be related to $\varepsilon_{0}(N)$ through (3.19). If we have $\left(u_{1, h}^{(n)}(x), u_{2, h}^{(n)}(x)\right) \in\langle\underline{\mathbf{u}}-\delta, \overline{\mathbf{u}}+\delta\rangle$ for all $x \in \bar{\Omega}$, then

$$
\begin{aligned}
& \left\|e_{i, h}^{(n+1)}\right\|_{C^{0, \beta}(\bar{\Omega})} \\
& \quad \leq K_{\varepsilon_{0}(N)}^{\prime} h^{\frac{1}{2}-\varepsilon_{0}(N)}\left[\left\|\left(u_{1}^{(\infty)}, u_{2}^{(\infty)}\right)\right\|_{2, \Omega}+M_{\lambda} \theta_{\lambda}^{n}\left\|\left(e_{1}^{(0)}, e_{2}^{(0)}\right)\right\|_{0, \Omega}\right] \\
& \quad+\left(K_{1}^{\prime} M_{\lambda}+K_{\varepsilon_{0}(N)}^{\prime} h^{\frac{1}{2}-\varepsilon_{0}(N)}\right)\left\|\left(e_{1, h}^{(n)}, e_{2, h}^{(n)}\right)\right\|_{0, \Omega}, \\
& \quad\left\|\left(e_{1, h}^{(n+1)}, e_{2, h}^{(n+1)}\right)\right\|_{0, \Omega} \\
& \quad \leq K_{2} h^{2}\left[\left\|\left(u_{1}^{(\infty)}, u_{2}^{(\infty)}\right)\right\|_{2, \Omega}+M_{\lambda} \theta_{\lambda}^{n}\left\|\left(e_{1}^{(0)}, e_{2}^{(0)}\right)\right\|_{0, \Omega}\right] \\
& \quad+\left(\theta_{\lambda}+K_{0}^{\prime} M_{\lambda} h^{2}\right)\left\|\left(e_{1, h}^{(n)}, e_{2, h}^{(n)}\right)\right\|_{0, \Omega},
\end{aligned}
$$

for $i=1,2,0<h \leq h_{0}$, for some $K_{1}^{\prime}>0, K_{2}>0$ independent of $n$ and $h$.

Proof. Cf. [5, Cor. 4.8].

(E6) Let $N=2$ or 3 . Choose $\bar{h}_{0}>0$ so small that

$$
0<\theta_{\lambda}+K_{2} M_{\lambda} \bar{h}_{0}^{2} \leq \rho_{\lambda}<1,
$$

for some $\rho_{\lambda}=\rho_{\lambda_{1}, \lambda_{2}}, 0<\rho_{\lambda}<1$. Then

$$
\begin{aligned}
& \left(u_{1, h}^{(n)}(x), u_{2, h}^{(n)}(x)\right) \in\langle\underline{\mathbf{u}}-\delta, \overline{\mathbf{u}}+\delta\rangle, \quad \forall x \in \bar{\Omega}, \\
& i=1,2, \quad n=1,2, \ldots, 0<h \leq \bar{h}_{0},
\end{aligned}
$$

and there exist $\bar{K}_{\varepsilon_{0}(N)}>0, \bar{K}_{0}>0$ such that

$$
\begin{gathered}
\left\|e_{i, h}^{(n+1)}\right\|_{C^{0, \beta}(\bar{\Omega})} \leq \bar{K}_{\varepsilon_{0}(N)} h^{\frac{1}{2}-\varepsilon_{0}(N)}\left[\left\|\left(u_{1}^{(\infty)}, u_{2}^{(\infty)}\right)\right\|_{2, \Omega}+\rho_{\lambda}^{n+1} h^{2}\left\|\left(e_{1}^{(0)}, e_{2}^{(0)}\right)\right\|_{0, \Omega}\right], \\
\left\|\left(e_{1, h}^{(n+1)}, e_{2, h}^{(n+1)}\right)\right\|_{0, \Omega} \leq \bar{K}_{0} h^{2}\left[\left\|\left(u_{1}^{(\infty)}, u_{2}^{(\infty)}\right)\right\|_{2, \Omega}+\rho_{\lambda}^{n+1} h^{2}\left\|\left(e_{1}^{(0)}, e_{2}^{(0)}\right)\right\|_{0, \Omega}\right],
\end{gathered}
$$

for $n=0,1,2, \ldots, 0<h \leq \bar{h}_{0}$.

Proof. A direct consequence of (3.21), (3.22) and (E5).

4. Comparison of eigenvalues And $\ell^{2}$-NORMS of $2 \times 2$ MAtrices

We now provide the rudiments of $2 \times 2$ matrices which form the basis in the formulation of the critical condition $\left[H_{2 \times 2}^{2}\right]$ in \$2

Let

$$
A=\left[\begin{array}{ll}
a & b \\
c & d
\end{array}\right]
$$

be a real $2 \times 2$ constant matrix. Then the $\ell^{2}$-norm of $A$ is defined by

$$
\|A\|=\sup _{\substack{x \in \mathbb{R}^{2} \\ x \neq 0}} \frac{|A x|}{|x|}, \quad|x|=\sqrt{x_{1}^{2}+x_{2}^{2}} .
$$

The next theorem gives an explicit characterization of $\|A\|$ in terms of its entries $a, b, c, d$.

Theorem 4.1. Let $A$ be given as in (4.1). Then

$$
\|A\|=\frac{1}{2}\left[\sqrt{(a+d)^{2}+(b-c)^{2}}+\sqrt{(a-d)^{2}+(b+c)^{2}}\right] .
$$


Proof. It is well known that (see, e.g., [13. p. 766])

$$
\|A\|=\max \left\{\sqrt{\mu_{1}}, \sqrt{\mu_{2}}\right\} \text {, where } \mu_{1}, \mu_{2}=\text { eigenvalues of } A^{T} A .
$$

Since

$$
A^{T} A=\left[\begin{array}{ll}
a^{2}+c^{2} & a b+c d \\
a b+c d & b^{2}+d^{2}
\end{array}\right]
$$

we get the characteristic polynomial of $A^{T} A$ :

$$
\mu^{2}-\left(a^{2}+b^{2}+c^{2}+d^{2}\right) \mu+\left[\left(a^{2}+c^{2}\right)\left(b^{2}+d^{2}\right)-(a b+c d)^{2}\right]=0 .
$$

Therefore

$$
\begin{aligned}
\mu_{1}, \mu_{2}= & \frac{1}{2}\left\{\left(a^{2}+b^{2}+c^{2}+d^{2}\right)\right. \\
& \left. \pm \sqrt{\left(a^{2}+b^{2}+c^{2}+d^{2}\right)^{2}-4\left[\left(a^{2}+c^{2}\right)\left(b^{2}+d^{2}\right)-(a b+c d)^{2}\right]}\right\} \\
= & \frac{1}{4}\left[\sqrt{(a+d)^{2}+(b-c)^{2}} \pm \sqrt{(a-d)^{2}+(b+c)^{2}}\right]^{2}
\end{aligned}
$$

from which we obtain

$$
\max \left\{\sqrt{\mu_{1}}, \sqrt{\mu_{2}}\right\}=\frac{1}{2}\left[\sqrt{(a+d)^{2}+(b-c)^{2}}+\sqrt{(a-d)^{2}+(b+d)^{2}}\right] .
$$

By (4.4), we have proved (4.3).

Theorem 4.2. Let

$$
D=\left[\begin{array}{cc}
\eta_{1} & 0 \\
0 & \eta_{2}
\end{array}\right], \quad \eta_{i}>0, \quad i=1,2,
$$

be a diagonal matrix, and let $x, y \in \mathbb{R}^{2}$ satisfy

$$
D x=A y, \quad \text { A given by 4.1). }
$$

Then

$$
|x| \leq \theta|y|,
$$

where

$$
\theta \equiv \frac{1}{2}\left[\sqrt{\left(\frac{a}{\eta_{1}}+\frac{d}{\eta_{2}}\right)^{2}+\left(\frac{b}{\eta_{1}}-\frac{c}{\eta_{2}}\right)^{2}}+\sqrt{\left(\frac{a}{\eta_{1}}-\frac{d}{\eta_{2}}\right)^{2}+\left(\frac{b}{\eta_{1}}+\frac{c}{\eta_{2}}\right)^{2}}\right] .
$$

Proof. From (4.6), we get

$$
x=D^{-1} A y=\left[\begin{array}{ll}
a / \eta_{1} & b / \eta_{1} \\
c / \eta_{2} & d / \eta_{2}
\end{array}\right] y \equiv \tilde{A} y .
$$

Applying Theorem 4.1 to $\tilde{A}$, we get (4.7).

We now consider a special form of the relation (4.6), where

$$
\begin{aligned}
D & =\left[\begin{array}{cc}
\lambda_{1}+\lambda_{1}^{*} & 0 \\
0 & \lambda_{2}+\lambda_{2}^{*}
\end{array}\right], \\
A & =\left[\begin{array}{cc}
\lambda_{1}-a & -b \\
-c & \lambda_{2}-d
\end{array}\right],
\end{aligned}
$$

where

$$
\left.\begin{array}{l}
\lambda_{1}^{*} \geq 0, \lambda_{2}^{*} \geq 0 ; \quad a, b, c, d \in \mathbb{R} ; \quad \lambda_{1}^{*}, \lambda_{2}^{*}, a, b, c \text { and } d \text { are given; } \\
\lambda_{1} \geq 0, \lambda_{2} \geq 0 ; \quad \lambda_{1}+\lambda_{1}^{*}>0, \lambda_{2}+\lambda_{2}^{*}>0 .
\end{array}\right\}
$$


Let $x, y \in \mathbb{R}^{2}$ satisfy

$$
D x=A y .
$$

Then by Theorem 4.2, we get

$$
|x| \leq\left\|D^{-1} A\right\||y| \equiv \theta_{\lambda}|y|
$$

where

$$
\begin{aligned}
\theta_{\lambda}=\frac{1}{2}\{ & {\left[\left(\frac{\lambda_{1}-a}{\lambda_{1}+\lambda_{1}^{*}}+\frac{\lambda_{2}-d}{\lambda_{2}+\lambda_{2}^{*}}\right)^{2}+\left(-\frac{b}{\lambda_{1}+\lambda_{1}^{*}}+\frac{c}{\lambda_{2}+\lambda_{2}^{*}}\right)^{2}\right]^{1 / 2} } \\
+ & {\left.\left[\left(\frac{\lambda_{1}-a}{\lambda_{1}+\lambda_{1}^{*}}-\frac{\lambda_{2}-d}{\lambda_{2}+\lambda_{2}^{*}}\right)^{2}+\left(\frac{b}{\lambda_{1}+\lambda_{1}^{*}}+\frac{c}{\lambda_{2}+\lambda_{2}^{*}}\right)^{2}\right]^{1 / 2}\right\} . }
\end{aligned}
$$

We want to investigate whether there exist any $\lambda_{1} \geq 0, \lambda_{2} \geq 0$, such that $0<\theta_{\lambda}<$ 1 , so that (4.13) becomes a contraction.

Theorem 4.3. Assume that (4.9)-(4.12) hold. If

$$
\lambda_{1}^{*}+a>0, \quad \lambda_{2}^{*}+d>0,
$$

then there exist some $\lambda_{1}>0, \lambda_{2}>0$, sufficiently large, such that $0<\theta_{\lambda}<1$, and (4.13) is a strict contraction.

Proof. Write $\theta_{\lambda}$ in (4.14) into the following form:

$$
\theta_{\lambda}=\frac{1}{2}\left\{\left[(\alpha+\delta)^{2}+(-\beta+\gamma)^{2}\right]^{1 / 2}+\left[(\alpha-\delta)^{2}+(\beta+\gamma)^{2}\right]^{1 / 2}\right\},
$$

where

$$
\alpha=\frac{\lambda_{1}-a}{\lambda_{1}+\lambda_{1}^{*}}, \quad \delta=\frac{\lambda_{2}-d}{\lambda_{2}+\lambda_{2}^{*}}, \quad \beta=\frac{b}{\lambda_{1}+\lambda_{1}^{*}}, \quad \gamma=\frac{c}{\lambda_{2}+\lambda_{2}^{*}} .
$$

Note that $\beta$ and $\gamma$ can be made arbitrarily small by taking $\lambda_{1}$ and $\lambda_{2}$ large. Therefore for large $\lambda_{1}>0$ and $\lambda_{2}>0$, we have $\theta_{\lambda}<1$ if and only if

$$
\frac{1}{2}\left\{\left[(\alpha+\delta)^{2}\right]^{1 / 2}+\left[(\alpha-\delta)^{2}\right]^{1 / 2}\right\}<1,
$$

i.e.,

$$
\frac{1}{2}[|\alpha+\delta|+|\alpha-\delta|]<1
$$

But for $\lambda_{1}>0$ and $\lambda_{2}>0$ sufficiently large, $\alpha$ and $\delta$ are positive, and

$$
\frac{1}{2}[|\alpha+\delta|+|\alpha-\delta|]= \begin{cases}\alpha & \text { if } \alpha \geq \delta, \\ \delta & \text { if } \alpha<\delta .\end{cases}
$$

Hence for large $\lambda_{1}$ and $\lambda_{2}$, if both $\alpha<1$ and $\delta<1$, then $\theta_{\lambda}<1$. But from (4.16) $\alpha<1$ and $\delta<1$ mean that

$$
\frac{\lambda_{1}-a}{\lambda_{1}+\lambda_{1}^{*}}<1, \quad \frac{\lambda_{2}-d}{\lambda_{2}+\lambda_{2}^{*}}<1,
$$

which hold if and only if

$$
\lambda_{1}^{*}+a>0 \quad \text { and } \quad \lambda_{2}^{*}+d>0 .
$$

We now consider the converse of Theorem 4.3. 
Theorem 4.4. Assume that (4.9)-(4.11) hold. If

$$
\lambda_{1}^{*}+a<0, \quad \lambda_{2}^{*}+d<0,
$$

then

$$
\theta_{\lambda} \equiv\left\|D^{-1} A\right\|>1
$$

Proof. From (4.17), we get

$$
-a>\lambda_{1}^{*}, \quad-d>\lambda_{2}^{*}
$$

Therefore, for $\lambda_{1} \geq 0, \lambda_{2} \geq 0$,

$$
\frac{\lambda_{1}-a}{\lambda_{1}+\lambda_{1}^{*}}>1, \quad \frac{\lambda_{2}-d}{\lambda_{2}+\lambda_{2}^{*}}>1
$$

From (4.14), we easily see that $\theta_{\lambda}>1$.

Is it still possible to have $0<\theta_{\lambda}<1$ if $\left(\lambda_{1}^{*}+a\right)\left(\lambda_{2}^{*}+d\right) \leq 0$ ? The answer is also negative, as given by the following.

Theorem 4.5. Assume (4.9)-(4.11), and

$$
\left(\lambda_{1}^{*}+a\right)\left(\lambda_{2}^{*}+d\right) \leq 0 .
$$

Then

$$
\theta_{\lambda} \equiv\left\|D^{-1} A\right\| \geq 1
$$

Proof. Condition (4.18) implies one of the following four possibilities:

(i) $\lambda_{1}^{*}+a=0$;

(ii) $\lambda_{2}^{*}+d=0$;

(iii) $\lambda_{1}^{*}+a<0, \lambda_{2}^{*}+d>0$;

(iv) $\lambda_{1}^{*}+a>0, \lambda_{2}^{*}+d<0$.

Cases (i) and (ii) are similar, as are (iii) and (iv). Here we only prove (iii). From $\lambda_{1}^{*}+a<0$, utilizing the facts that $\lambda_{1} \geq 0, \lambda_{1}^{*} \geq 0$ and $\lambda_{1}+\lambda_{1}^{*}>0$, we have

$$
\frac{\lambda_{1}-a}{\lambda_{1}+\lambda_{1}^{*}}>1
$$

From (4.14), we get

$$
\theta_{\lambda} \geq \frac{1}{2}\left\{\left|\frac{\lambda_{1}-a}{\lambda_{1}+\lambda_{1}^{*}}+\frac{\lambda_{2}-d}{\lambda_{2}+\lambda_{2}^{*}}\right|+\left|\frac{\lambda_{1}-a}{\lambda_{1}+\lambda_{1}^{*}}-\frac{\lambda_{2}-d}{\lambda_{2}+\lambda_{2}^{*}}\right|\right\}>1
$$

Remark 4.6. Theorems 4.34.5 show that 4.15) is a necessary and sufficient condition for $0<\theta_{\lambda}<1$. It is also interesting to note that the strict contraction property (4.13) does not depend on the off-diagonal entries $-b$ and $-c$ in the matrix $A$ in 44.10$)$. Since our critical condition $\left[H_{2 \times 2}^{2}\right]$ in $\$ 2$ was formulated on the basis of (4.15), we see that the strict contraction property (2.26) in Theorem 2.2 is independent of the "off-diagonal nonlinearities" in $\partial f_{1} / \partial u_{2}$ and $\partial f_{2} / \partial u_{1}$, for $f_{1}$ and $f_{2}$ given in (2.1). 


\section{Numerical Example}

We construct an annular domain $\Omega$ as shown in Figure 5.1 wherein $\partial \Omega$ consists of an exterior bounding circle $C_{1}$ and an interior nonconcentric bounding circle $C_{2} . C_{1}$ and $C_{2}$ in Figure 5.1 are discretized uniformly into, respectively, 96 and 48 panels which are piecewise constant boundary elements, as indicated in Figure 5.1. Thus, what we have is an $S_{h}^{1,0}(\partial \Omega)$-system, which is also an $S_{h}^{d}$-system $(d=$ 0 ) of smoothest splines of degree 0 with a quasiuniform mesh. So Theorem 3.4 is applicable. For the volume potential (i.e., the domain integral) in (3.3), the numerical integration is achieved by placing 1620 Gaussian quadrature points on $\bar{\Omega}$, which are rather evenly distributed on $\bar{\Omega}$. Note that $\Omega$ is a nonconvex, nonstarshaped, doubly connected domain. The algorithmic flowchart is nearly the same as that in [5 \$7]. The computer programs on semilinear $2 \times 2$ system were first tested with synthetic data, and produced numerical solutions of high accuracy with strong trends of convergence.

We include a simple numerical example below.

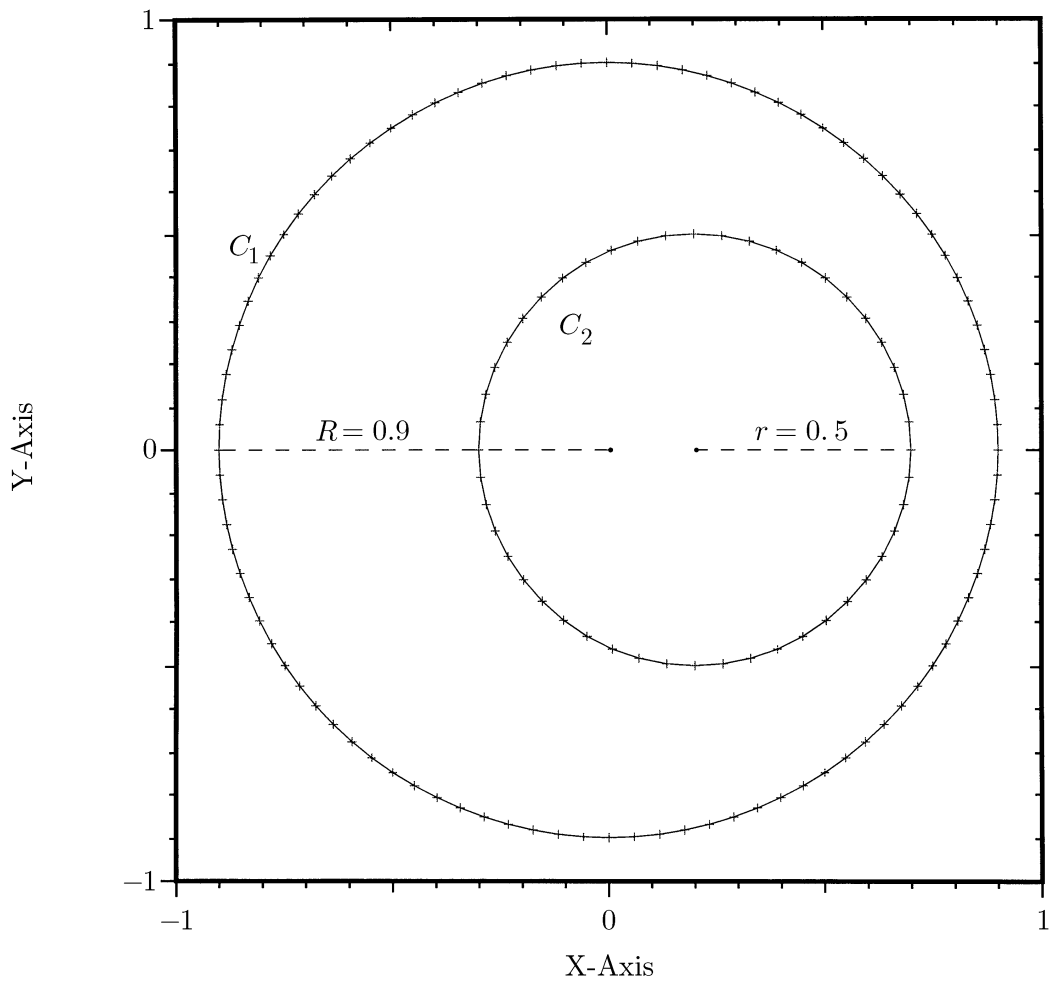

Figure 5.1. The domain $\Omega$ for Example [5.1; it is bounded by an exterior circle $C_{1}$ and an interior circle $C_{2}$. $C_{1}$ and $C_{2}$ are uniformly discretized, respectively, into 96 and 48 piecewise constant boundary elements. 
Example 5.1. A nonlinear monotone dissipative operator system that is mixed quasimonotone. Consider

$$
\begin{cases}\Delta u_{1}(x)=3\left[3 u_{1}^{3}(x)+2 u_{2}(x)\right]+\frac{15}{4} & \text { on } \Omega, \\ \Delta u_{2}(x)=3\left[-2 u_{1}(x)+3 u_{2}^{3}(x)\right]-6 & \text { on } \Omega, \\ u_{1}(x)=u_{2}(x)=0 & \text { on } \partial \Omega .\end{cases}
$$

Then

$$
\left\{\begin{array}{l}
f_{1}\left(x, u_{1}, u_{2}\right)=f_{1}\left(u_{1}, u_{2}\right)=3\left(3 u_{1}^{3}+2 u_{2}\right)+\frac{15}{4} \\
f_{2}\left(x, u_{1}, u_{2}\right)=f_{2}\left(u_{1}, u_{2}\right)=3\left(-2 u_{1}+3 u_{2}^{3}\right)-6
\end{array}\right.
$$

satisfy

$$
\frac{\partial f_{1}}{\partial u_{2}}=6>0, \quad \frac{\partial f_{2}}{\partial u_{1}}=-6<0 .
$$

Therefore (5.1) is mixed quasimonotone.

Define a nonlinear operator

$$
\mathcal{N}\left(\left[\begin{array}{l}
u_{1} \\
u_{2}
\end{array}\right]\right)=\left[\begin{array}{c}
\Delta u_{1}-3\left(3 u_{1}^{3}+2 u_{2}\right) \\
\Delta u_{2}-3\left(-2 u_{1}+3 u_{2}^{3}\right)
\end{array}\right] .
$$

Then

$$
\begin{aligned}
& \left\langle\mathcal{N}\left(\left[\begin{array}{l}
u_{1} \\
u_{2}
\end{array}\right]\right)-\mathcal{N}\left(\left[\begin{array}{l}
v_{1} \\
v_{2}
\end{array}\right]\right),\left[\begin{array}{l}
u_{1} \\
u_{2}
\end{array}\right]-\left[\begin{array}{l}
v_{1} \\
v_{2}
\end{array}\right]\right\rangle \\
= & -\sum_{i=1}^{2} \int_{\Omega}\left[\left|\nabla\left(u_{i}-v_{i}\right)\right|^{2}+9\left(u_{i}^{3}-v_{i}^{3}\right)\left(u_{i}-v_{i}\right)\right] d x \leq 0,
\end{aligned}
$$

for $u_{i}, v_{i} \in H_{0}^{2}(\Omega)$. Therefore $\mathcal{N}$ is a monotone dissipative operator, and (5.1) admits a unique solution pair $\left(u_{1}, u_{2}\right)$ [7].

It is straightforward to check that

$$
\left(\underline{u}_{1}, \bar{u}_{1}\right)=(-1,0), \quad\left(\underline{u}_{2}, \bar{u}_{2}\right)=\left(0,\left(\frac{2}{3}\right)^{\frac{1}{3}}\right)
$$

form ordered pairs of constant subsolutions and supersolutions for $u_{1}$ and $u_{2}$, respectively. Also, since

$$
\frac{\partial f_{1}}{\partial u_{1}}=27 u_{1}^{2} \geq 0, \quad \frac{\partial f_{2}}{\partial u_{2}}=27 u_{2}^{2} \geq 0,
$$

we see that (2.40), and consequently $\left[H_{2 \times 2}^{2}\right]$, is trivially satisfied because $\lambda_{1}^{*}>$ 0 . Although condition (2.22) in Theorem [2.1] is violated, still, according to the monotone dissipative property of $\mathcal{N}$ stated after (5.1), it is not hard to modify the proof in [11, p. 409] to establish that the quasimonotone iterations (2.19) and (2.20) converge to the unique solution. Since $\left.\left[H_{2 \times 2}^{1}\right]\right]$ is also easily verifiable, Theorem 3.4 holds with $d=0$, and $0 \leq r<2$.

As with [5], the Galerkin approximation (3.10) is replaced by a point collocation scheme:

$$
\left\{\begin{array}{l}
\left(S_{\lambda} \eta_{j, h}^{(n+1)}\right)\left(x_{i}\right)=g_{j}\left(x_{i}\right)+\left(V_{\lambda} F_{1, \lambda, h}^{(n)}\right)\left(x_{i}\right), \quad j=1,2, \\
x_{i} \text { are the midpoints of the piecewise constant boundary elements, } \\
\text { for } 1 \leq i \leq M, \text { for some } M,
\end{array}\right.
$$

where only $(3.8)_{1}$ is used because the boundary conditions for both $u_{1}$ and $u_{2}$ are Dirichlet. We choose $\lambda\left(=\lambda_{1}=\lambda_{2}\right)=(\underline{5.4})^{2}$. Actually, any small to moderate size 
positive $\lambda$ will work. The point collocation scheme (5.6) should have the same type of accuracy as the Galerkin scheme (3.8), according to Arnold and Wendland [2].

The error estimate (3.11) contains two parameters, $h$ and $n$. We verify its convergence rates by the computer as follows:

(i) Fix $n=2$. Vary $h$ by doubling the number of meshes as follows:

$$
\begin{array}{llc}
h_{0}: & 48\left(\text { on } C_{1}\right)+24 & \left(\text { on } C_{2}\right) ; \\
h_{1}: & 96\left(\text { on } C_{1}\right)+48 & \left(\text { on } C_{2}\right) ; \\
h_{2}: & 192\left(\text { on } C_{1}\right)+96 & \left(\text { on } C_{2}\right) ; \\
h_{3}: & 384\left(\text { on } C_{1}\right)+192 & \left(\text { on } C_{2}\right)
\end{array}
$$

We then compute the logarithmic relative error

$$
\ln \left\|\left(\eta_{1, h_{j}}^{(2)}-\eta_{1, h_{j-1}}^{(2)}, \eta_{2, h_{j}}^{(2)}-\eta_{2, h_{j-1}}^{(2)}\right)\right\|_{0, \partial \Omega}, \quad j=1,2,3
$$

and plot them against $\ln h_{j}$. We obtain a line in Figure 5.2, whose slope is measured to be 0.496 . This is totally consistent with the rate $\mathcal{O}\left(h^{\frac{1}{2}}\right)$ (setting $s=0$ in (3.11)) given in Theorem 3.4 .

(ii) Fix $h=h_{2}$; see (5.7). We compute the logarithmic relative errors

$$
\ln \left\|\left(\eta_{1, h_{2}}^{(n)}-\eta_{1, h_{2}}^{(n-1)}, \eta_{2, h_{2}}^{(n)}-\eta_{2, h_{2}}^{(n-1)}\right)\right\|_{0, \partial \Omega}, \quad n=2,3,4,5,6,
$$

and plot them against $n$, yielding Figure 5.3 Again we have found that these five points lie neatly on a line, with a slope equal to -0.39 . This is totally consistent with the rate $\mathcal{O}\left(\rho_{\lambda}^{n}\right)$ in (3.11). In fact, it also tells that

$$
\ln \rho_{\lambda} \approx-0.39, \quad \text { i.e., } \quad \rho_{\lambda} \approx 0.677 \text {. }
$$

Finally, using $h=h_{3}$, see (5.7), and iterating 21 times, we obtain the approximate profiles of $u_{1}^{(\infty)}$ and $u_{2}^{(\infty)}$ given, respectively, in Figures 5.4 and 5.5 .

For this example, any negative constant $u_{1}^{(0)},-1<u_{1}^{(0)}<0$, can be used as an initial iterate for $u_{1}(\cdot)$, and any positive constant constant $u_{2}^{(0)}, 0<u_{2}^{(0)}<\left(\frac{2}{3}\right)^{\frac{1}{3}}$, can be used as an initial iterate for $u_{2}(\cdot)$.

Many other examples, such as the steady state Lotka-Volterra system of predatorprey model in Pao [11, Ch. 8] and the population migration pattern as studied in Matano and Mimma [8], in principle can also be computed by quasimonotone iterations. However, we have not yet obtained totally satisfactory numerical results. The primary reason for this is due to the difficulty in choosing the specific upper and lower solutions associated with such problems (unlike Example 5.1, where such choices as constants can be easily made). Some further numerical experiments and improvements are needed. 


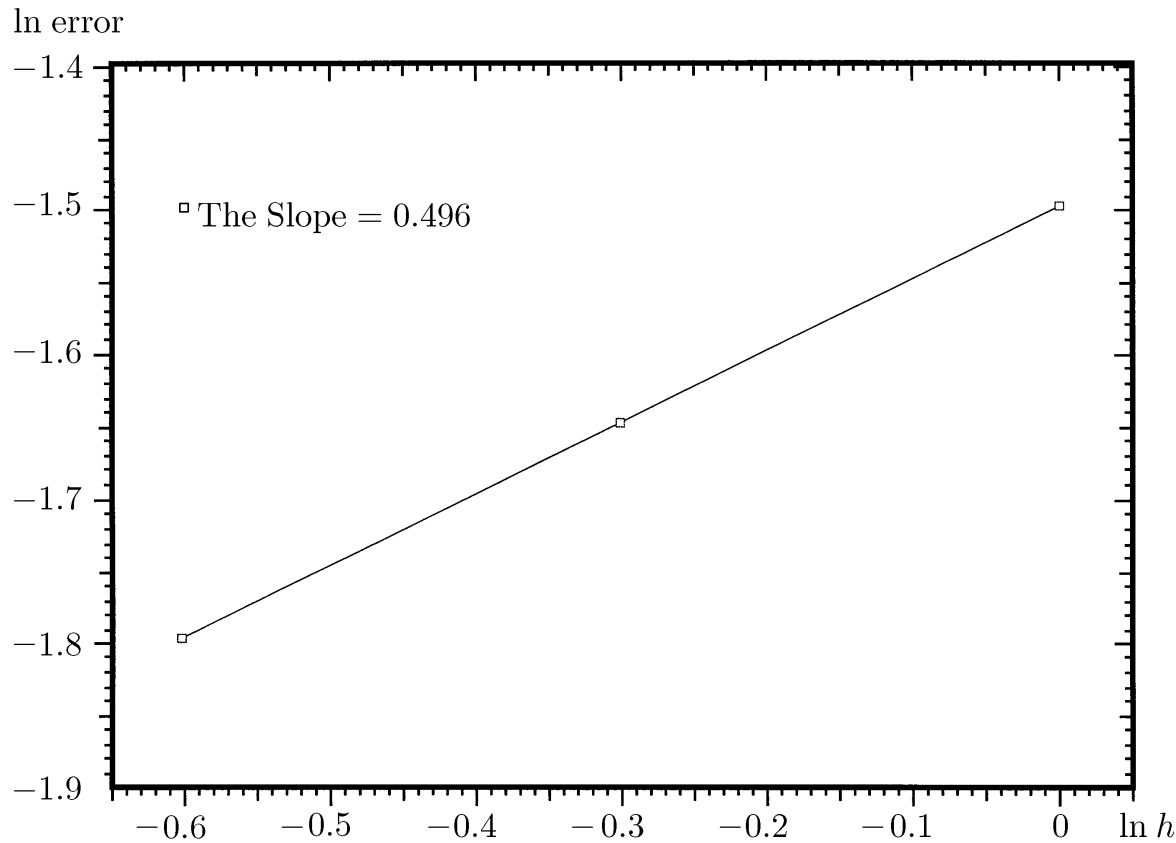

FiguRE 5.2. The relative convergence rate of

$$
\ln \left\|\left(\eta_{1, h}^{(2)}-\eta_{1,0.5 h}^{(2)}, \eta_{2, h}^{(2)}-\eta_{2,0.5 h}^{(2)}\right)\right\|_{L^{2}(\partial \Omega)}
$$

for Example 5.1 plotted against $\ln h$. The slope of the line, 0.496, is close to the theoretical estimate 0.5 , i.e., $\mathcal{O}\left(h^{\frac{1}{2}}\right)$.

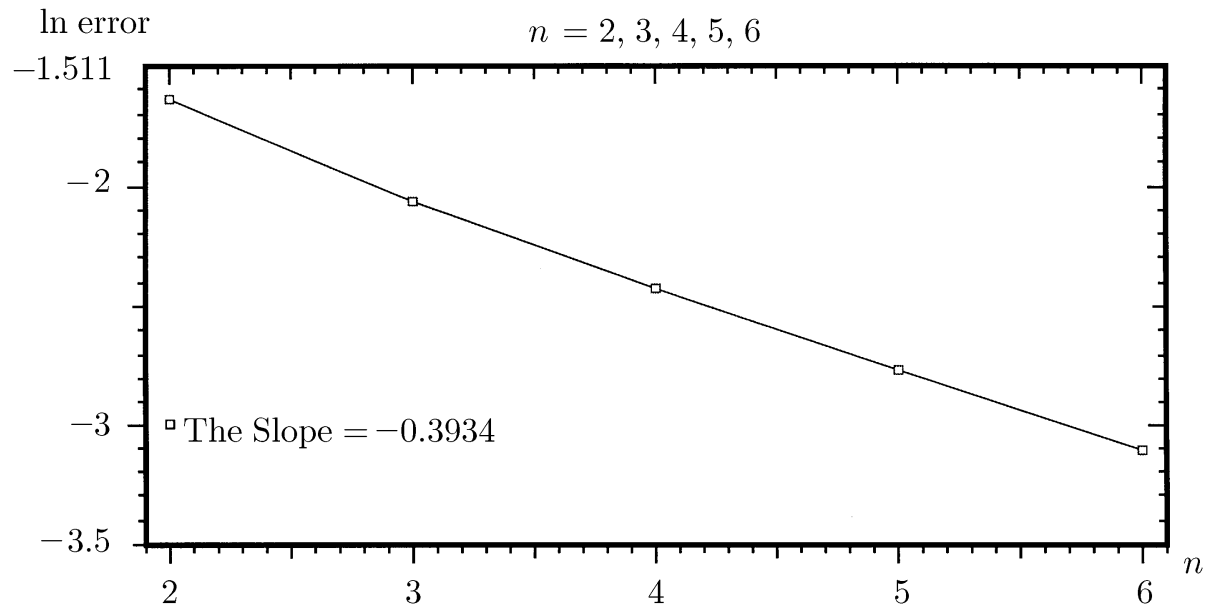

FiguRE 5.3. The relative convergence rate of

$$
\ln \left\|\left(\eta_{1, h}^{(n)}-\eta_{1, h}^{(n+1)}, \eta_{2, h}^{(n)}-\eta_{2, h}^{(n+1)}\right)\right\|_{L^{2}(\partial \Omega)}
$$

for Example 5.1 plotted against $n$ with $h$ being fixed. The points lie neatly on a line, consistent with the theoretical estimate $\mathcal{O}\left(\rho_{\lambda}^{n}\right)$. 


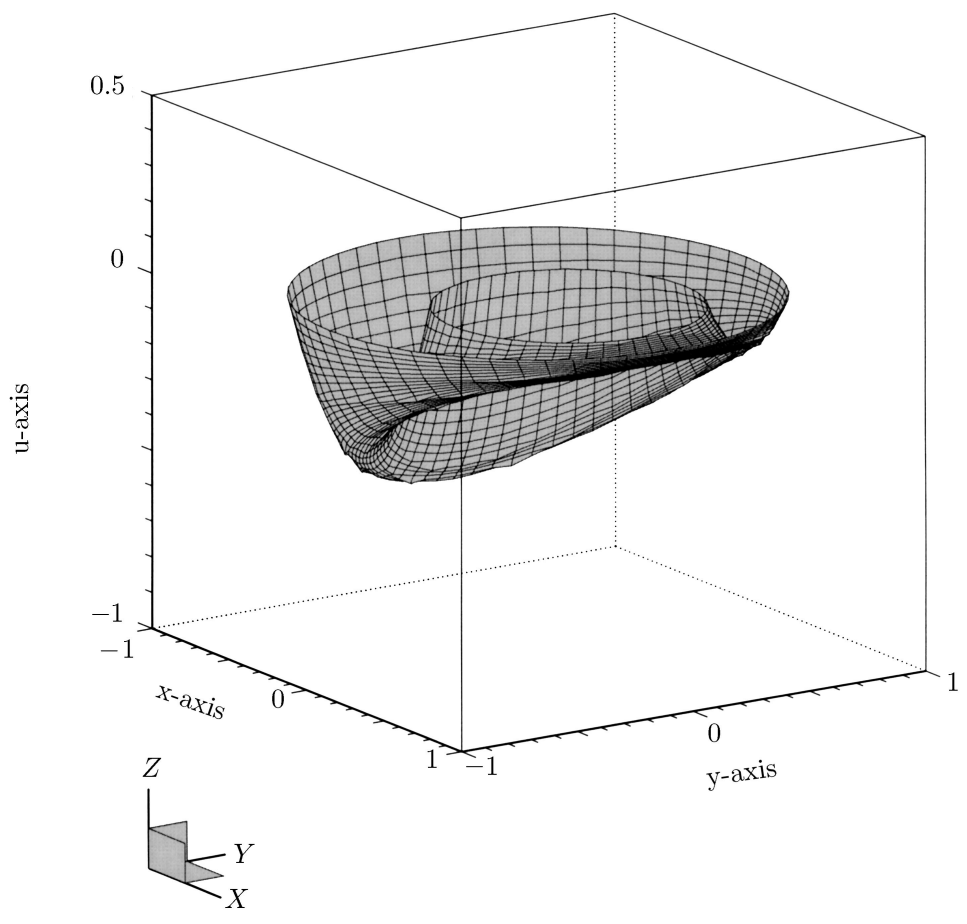

Figure 5.4. The profile of $u_{1}$ for Example 5.1.

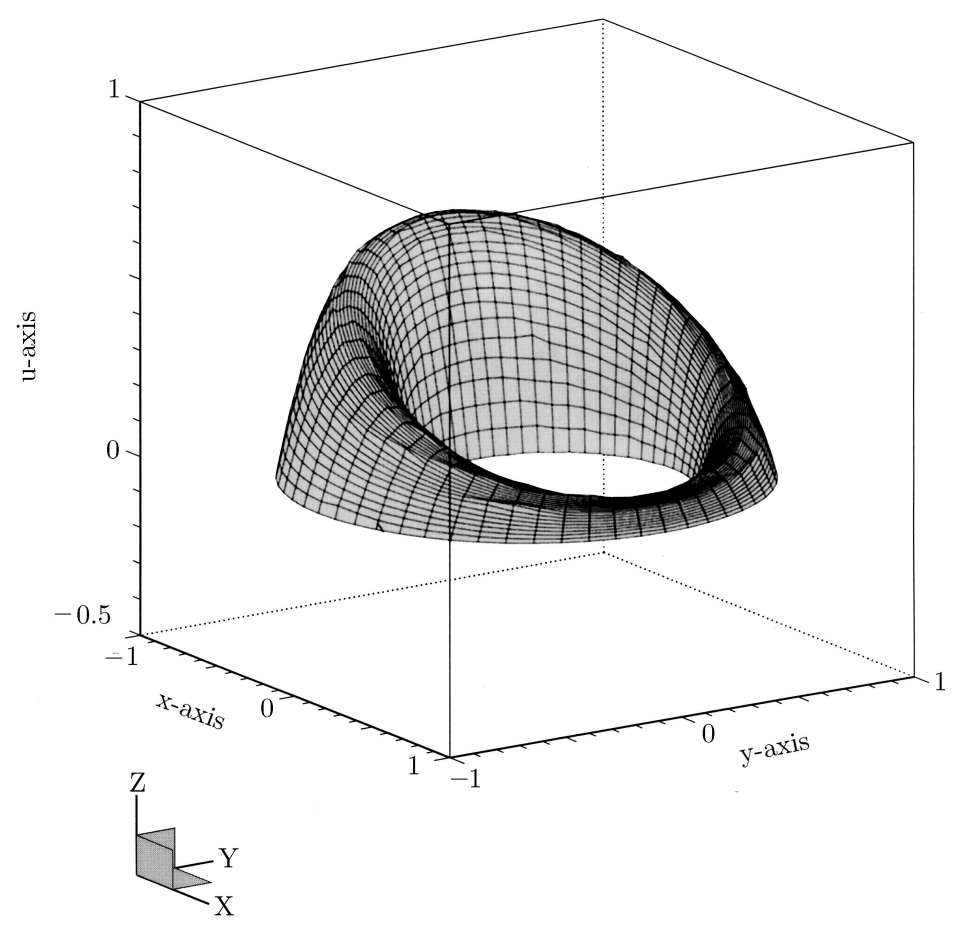

Figure 5.5. The profile of $u_{2}$ for Example 5.1. 


\section{ACKNOWLEDGEMENTS}

All of the computations were carried out on an SGI Indigo 2 Extreme Graphics workstation provided to us via NSF Grant DMS 94-04380, Dr. John E. Lagnese, Program Manager. Our grateful appreciation is herewith acknowledged.

\section{REFERENCES}

[1] M. Abramowitz and I.A. Stegun, Handbook of Mathematical Functions, Dover, New York, 1966. MR 34:8606

[2] D.N. Arnold and W.L. Wendland, The convergence of spline collocation for strongly elliptic equations on curves, Numer. Math. 47 (1985), 317-341. MR 87f:65142

[3] I. Babuska and A.K. Aziz, Survey lectures on the mathematical foundations of the finite element method, The Mathematical Foundations of the Finite Element Method with Applications to Partial Differential Equations, Academic Press, New York, 1972, pp. 1-359. MR 54:9111

[4] G. Chen and J. Zhou, Boundary Element Methods, Academic Press, London-San Diego, 1992. MR 93e:65143

[5] Y. Deng, G. Chen, W.M. Ni and J. Zhou, Boundary element monotone iteration scheme for semilinear elliptic partial differential equations, Math. Comp. 65 (1996), 943-982. MR 66j:65134

[6] G.C. Hsiao and W.L. Wendland, The Aubin-Nitsche lemma for integral equations, J. Integral Eq. 3 (1981), 299-315. MR 83j:45019

[7] J.L. Lions, Quelques Methodes de Resolution des Problemes aux Limites Non Lineaires, Dunod, Paris, 1969. MR 41:4326

[8] H. Matano and M. Mimura, Pattern formation in competition-diffusion systems in nonconvex domain, Publ. RIMS, Kyoto Univ. 19(1983), 1049-1079. MR 85e:35065

[9] W.M. Ni, Some aspects of semilinear elliptic equations, Lecture Notes published by Institute of Mathematics, National Tsing Hua Univ., Hsinchu, Taiwan, Rep. of China, May, 1987.

[10] C.V. Pao, Numerical solutions for some coupled systems of nonlinear boundary value problems, Numer. Math. 51 (1987), 381-394. MR 89f:65115

[11] C.V. Pao, Nonlinear Parabolic and Elliptic Equations, Plenum Press, New York, 1992. MR 94c:35002

[12] K. Ruotsalainen and J. Saranen, On the convergence of the Galerkin method for nonsmooth solutions of integral equations, Num. Math. 54(1988), 295-302. MR 90a:65281

[13] D.M. Young and R.T. Gregory, A Survey of Numerical Mathematics, Vol. II, Addison-Wesley, Reading, MA, 1973. MR 53:11954

Department of Mathematics, Texas A\&M University, College Station, TX 77843

E-mail address: gchen@math.tamu.edu

E-mail address: jzhou@math.tamu.edu

Northern Telecom, 2201 Lakeside Blvd, Richardson, Texas 75082

E-mail address: ydeng@nortel.ca

School of Mathematics, University of Minnesota, Minneapolis, MN 55455

E-mail address: ni@math.umn.edu 